\title{
Request for Approval Vented Container Annual Release Fraction
}

\section{RECORD COPY}

United States

Department of Energy

Richland, Washington 


\section{Request for Approval Vented Container Annual Release Fraction}

Date Published

September 1999

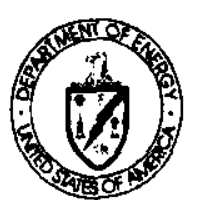

\section{United States}

Department of Energy

P.o. Box 550

Richland, Washington

RECORD COPY

Approved for Public Release 


\section{RELEASE AUTHORIZATION}

Document Number: $\quad$ DOE/RL-99-60

Document Title: Fraction

This document, reviewed in accordance with DOE Order 241.1, "Scientific and Technical Information Management," and 241.1-1. "Guide to the Management of Scientific and Technical Information," does not contain classified or sensitive unclassified information and is:

APPROVED FOR PUBLIC RELEASE

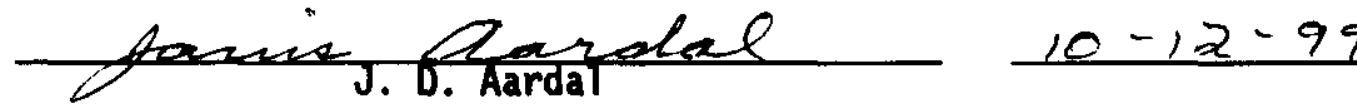

Lockheed Martin Services, Inc. Document Control/Information Clearance

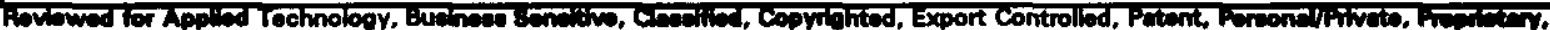

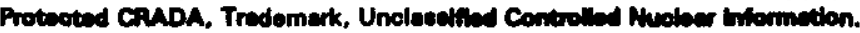

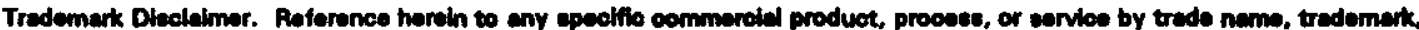

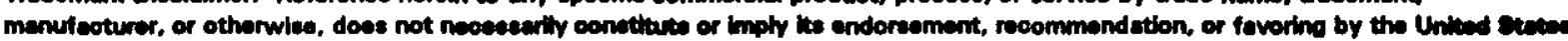

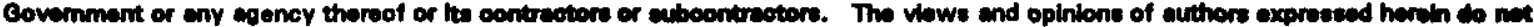

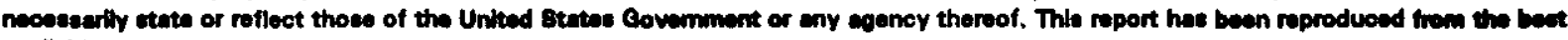
averieble copy.

Printed in the United States of Amorios.

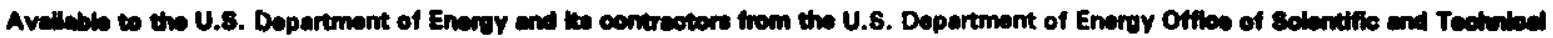
intermedion, P.O. Box 62, Oak Aldes, TN 37031; Telephone: 423576-8401.

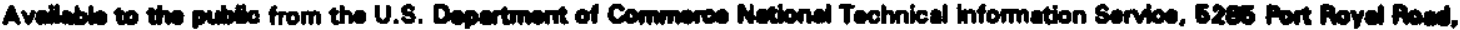
Spoingfield, VA 22181; Tolophone: 703/4e7-4050. 


\section{CONTENTS}

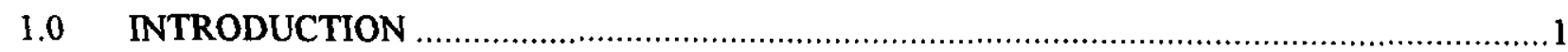

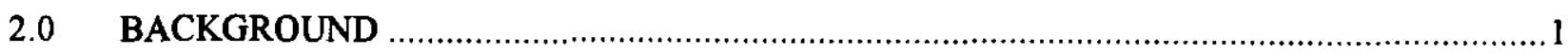

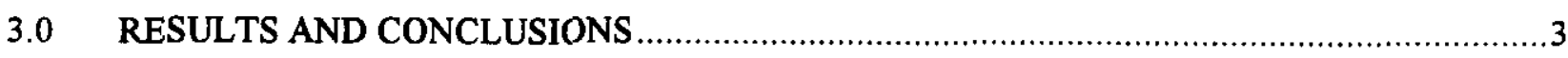

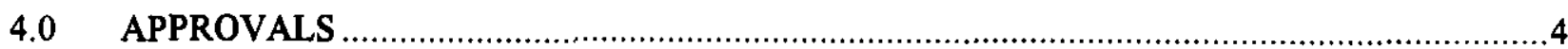

\section{APPENDICES}

A ANALYTICAL RESULTS ON TWELVE NUCFIL ${ }^{\circledR}$ FILTER SAMPLES RECEIVED IN JUNE 1999 APP A-i

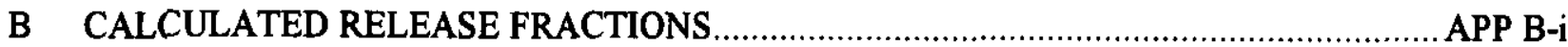




\section{REQUEST FOR APPROVAL VENTED CONTAINER ANNUAL RELEASE FRACTION}

\subsection{INTRODUCTION}

In accordance with the approval conditions for Modification to the Central Waste Complex (CWC) Radioactive Air Emissions Notice of Construction (NOC), dated August 24, 1998, a new release fraction has been developed for submittal to the Washington State Department of Health (WDOH). The proposed annual release fraction of $2.50 \mathrm{E}-14$ is proposed for use in future NOCs involving the storage and handling operations associated with vented containers on the Hanford Site. The proposed annual release fraction was the largest release fraction calculated from alpha measurements of the $\mathrm{NucFil}^{\oplus}$ filters from 10 vented containers consisting of nine 55-gallon drums and one burial box with dimensions of $9.3 \times 5.7$ x 6.4 feet. An annual release fraction of 2.0 E-09 was used in the modification to the CWC radioactive air emissions NOC. This study confirmed that the release fraction used in the CWC radioactive air emissions NOC was conservative.

\subsection{BACKGROUND}

On September 21, 1998, a meeting was held between the U.S. Department of Energy, Richland Operations Office and WDOH. Based on questions asked by WDOH, it was determined that the 222-S Laboratory Complex lowest detection limits for alpha decaying radionuclides were in the range of 0.75 to 1.5 picocuries. Detection limits for beta and gamma decaying radionuclides were in the range of 15 to 150 picocuries and 50 to 100 picocuries, respectively. Vented containers within CWC were selected based on having a high alpha radionuclide content yet still meeting the transportation and $T$ Plant Complex radiological/criticality safety limits. In addition, all containers selected have been handled and stored for at least 3 years. Selecting vented containers with a high alpha radionuclide content and long storage times combined with the availability of low detection capabilities for alpha decay provided the best opportunity to calculate an actual annual release fraction. The equation for calculating release fractions proposed at the September 21, 1998, meeting with WDOH, was modified to include the fraction of spike actually recovered. This will result in a more accurate calculated release fraction. For conservatism, the fraction of spike material actually recovered was only used when the value was less than one. The vented container annual release fractions were calculated using the following formula:

$$
R F=\frac{\frac{\text { Count }}{0.9995 \times S R}}{\text { Inventory } \times \text { Time }}
$$

Where:

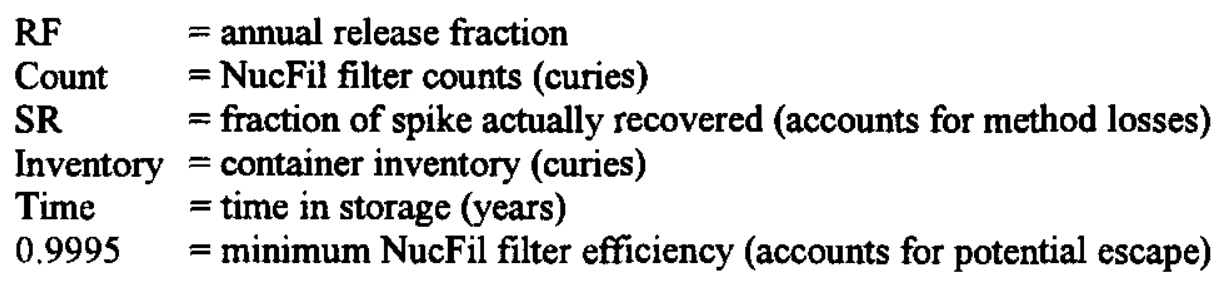

(B) NucFil is a registered trademark of Nuclear Filter Technology, Incorporated, Golden, Colorado. 
Reference: Radioactive Air Emissions Program Notice of Construction for the Central Waste Complex and Enhance Radioactive and Mixed Waste Storage, Phase V, Project $W-112$, DOE/RL-95-79, dated August 1995.

NucFil filters were prepared for sample analysis as described in a summary of the laboratory report contained within Appendix A. A spiked and a blank sample were prepared from two unused NucFil filters. The spike sample was prepared using cesium (Cs-137) and americium (Am-241). Cs-137 was selected as the gamma emitter, barium (Ba-137) a decay product of $\mathrm{Cs}-137$ was the beta emitter, and Am241 was selected as the alpha emitter.

The selected containers and the general description of the container contents are as follows.

Container identification

WH-86-034

(55-gallon drum)

BP-189055

(55-gallon drum)

BP-192018

(55-gallon drum)

BP-192019

(55-gallon drum)

RH-A-90-005

(55-gallon drum)

RH-A-92-006

(55-gallon drum)

RH-A-95-012/9408762

(55-gallon drum)

RH-A-95-013/9406626

(55-gallon drum)

BP-189047

(55-gallon drum)

PNL-TRU-95018

(burial box)
General description of container contents

Metal, cement, chemicals, absorbent, plastic and 10 mil liner

Absorbent, asbestos, cloth, glass, metal, paper/cardboard, Plastic/polyurethane, and lead

10 mil liner, absorbent, glass, and metal

10 mil liner, absorbent, cloth, dirt, glass, metal, paper/cardboard, Plastic/polyurethane, and nubber

Absorbent, cloth, glass, metal, paper/cardboard, Plastic/polyurethane, wood, and rubber

Absorbent, cloth, and plastic

Absorbent, anti-corrosive radpad, cloth, metal, plastic/polyurethane, and rubber

Absorbent, anti-corrosive radpad, cloth, metal, plastic/polyurethane, and rubber

Absorbent, ceramics, glass, liquid, metal, paper/cardboard, plastic/polyurethane, and rubber

Cloth, floor sweepings, glass, metal, paper/cardboard, plastic, rubber, and wood

Operational experience within the Central Waste Complex (e.g., frequent visual inspections and radiation surveys of the containers) has demonstrated that the containers are sound and that external contamination does not exist on the exterior of the containers. Any release of radionuclides would be through the NucFil Filters. 


\subsection{RESULTS AND CONCLUSIONS}

Appendix B contains a summary of the results for both alpha and beta/gamma counts for each NucFil filter and the calculated annual release fraction. Because of the low sample counts, the blank was not subtracted from the actual results because many of the samples were lower than the blank, which would result in the calculation of negative annual release fractions.

For alpha decaying radionuclides, six of the sample results were reported as less-than values. For computational purposes, the number reported in the less-than value was used as the actual value for calculating an annual release fraction. Samples that were not reported as less-than values were at or above the blank value. The calculated release fractions based on alpha detection ranged from 3.23 E-15 to $2.50 \mathrm{E}-14$. As noted in Appendix A, the $500 \%$ error associated with the count used to calculate the highest release fraction of $2.50 \mathrm{E}-14$ was attributed to the sample count being less than background.

For beta decay measurements, only one of the sample results was reported as a less-than value. For computational purposes, the number reported in the less-than value also was used as the actual value for calculating an annual release fraction. As mentioned in Appendix A, there were no gamma emitters detected. The beta/gamma numbers were reported together because the inventory within the vented containers is tracked based on the sum of the beta and gamma radionuclides. The calculated annual release fractions based on beta/gamma detection ranged from $2.33 \mathrm{E}-11$ to $6.98 \mathrm{E}-09$.

With the exception of three samples, all of the samples were less than the blank, which suggests that the detected beta activity occurring in the blank might be from naturally occurring beta decay within the charcoal located inside the NucFil filter (e.g., carbon-14 and/or potassium-40). The release fractions calculated for the beta/gamma isotopes are unreliable and were reported only for completeness. The calculated annual release fractions are considered unreliable because of the low inventory of beta/gamma decaying radionuclides within the selected containers (1,000 to 10,000 times less than the inventory of alpha decaying radionuclides) and the presence of naturally occurring beta emitters within the charcoal located inside the NucFil filter. To obtain reliable release fractions for beta/gamma isotopes, containers with a high inventory of beta/gamma decaying radionuclides would need to be selected.

Drums with a high alpha activity were chosen over those with beta/gamma activity because a lower Practical Quantitation Limit could be achieved with alpha activity and the samples are more easily distinguished from the blank. Furthermore, a high loading of beta-gamma activity would have exposed workers to a significant radiation hazard. The annual release fraction of $2.0 \mathrm{E}-09$ used in the modification to the CWC radioactive NOC is much larger than the calculated $2.5 \mathrm{E}-14$ annual release fraction. 


\section{APPROVALS}
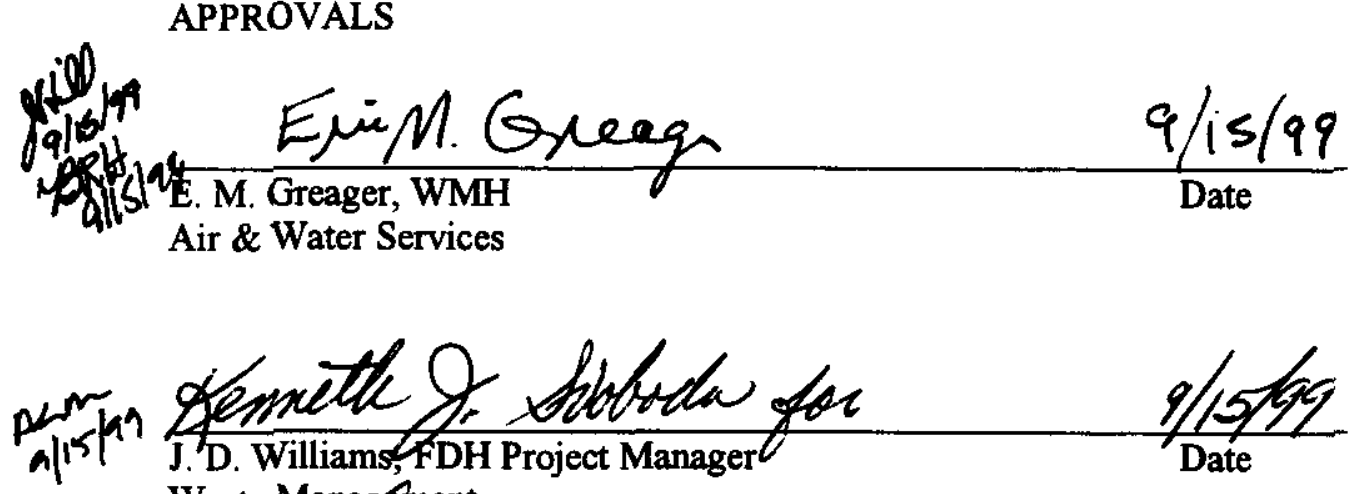

Waste Management
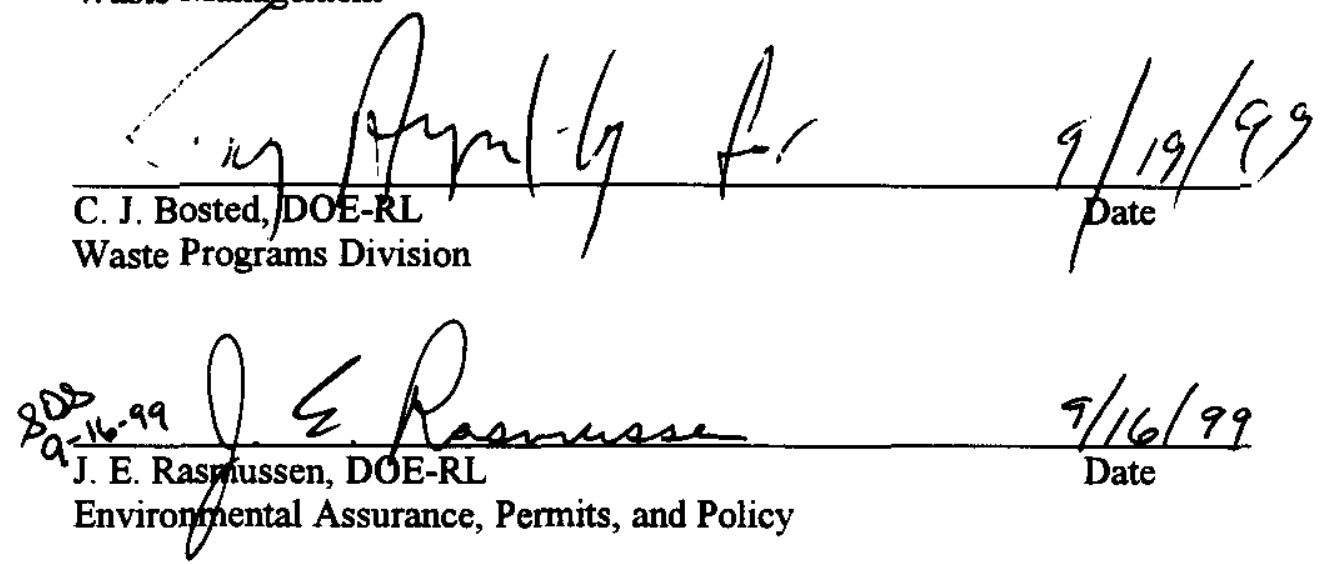

\begin{tabular}{l}
\hline A. W. Conklin, WDOH \\
Division of Radiation Protection, \\
Air Emissions
\end{tabular}


DOE/RL-99-60, Rev. 0 09/99

APPENDIX A

\section{ANALYTICAL RESULTS ON TWELVE NUCFL' ${ }^{\circledR}$ FILTER SAMPLES RECEIVED IN JUNE 1999}




\section{ANALYTICAL RESULTS ON TWELVE NUCFI ${ }^{\circledR}$ FILTER SAMPLES RECEIVED IN JUNE 1999}

This appendix discusses the analytical results on twelve filter samples received on June 28, 1999. The samples were analyzed for total alpha and total beta activities, and cobalt- 60 , niobium- 94 , ruthenium/rhodium-106, antimony-125, cesium-134, cesium-137, cerium/praseodynium-144, europium152, europium-154, europium-155, and americium-241 by gamma energy analysis (GEA). The remainder of the GEA library was also provided. A diagram indicating the sample breakdown and preparation procedures is included as Attachment 1. Tables containing all the analytical results are included at Attachment 2.

The samples were prepared and analyzed as described in the reference with one exception. The two $\mathrm{NucFil}^{\text {B }}$ filters received as sample 221T-99-035 were not composited before sample preparation and analysis. Both of the filters in sample 221T-99-035 were prepared in the same manner as the other samples. One of the filters was analyzed for total alpha, total beta, and GEA as the blank. Alpha and beta spikes were added to the other sample following digestion to access the efficiency of sample transfer, mounting, and counting. This sample was analyzed for total alpha and total beta activities.

Sample preparation took place as follows. The lid was removed from each sample with a handsaw. The carbon filter and silicone rubber sealant were removed with a surgical blade and placed in a beaker. The samples were ashed at approximately $550^{\circ} \mathrm{C}$. There was no visible remainder of the carbon filters, although the silicone rubber survived the ashing. The beakers and silicone rubber remainders were rinsed with a nitric acid solution that was transferred into a volumetric flask for GEA. Next, the solutions were mounted for alpha and beta counting. Hence, the entire sample was used for each analysis.

No radionuclides were detected by GEA in any of the samples, however only sample 221T-99-026 had no detectable alpha or beta activity. Total alpha and total beta activities were detected in samples $221 \mathrm{~T}$ $99-025,-032,-033,-034$, and -035 (the blank). Only total beta activity was detected in samples $221 \mathrm{~T}-99-$ $027,-028,-029,-030$, and -031 . The highest detected total alpha activity was $1.22 \times 10^{-6} \mathrm{uCi}$ found in sample 221T-99-034. The highest detected total beta activity was $3.59 \times 10^{-5} \mathrm{uCi}$ found in sample 221T. 99-030.

Each sample was counted once for total alpha and total beta and once for GEA. The total alpha, total beta, and GEA laboratory control standards were within the laboratory control limits. The total alpha and total beta spike recoveries were also within the laboratory control limits. The "spikes" were not true matrix spikes since the spikes were added to a blank as directed and not a sample. Nevertheless, the good spike recoveries indicated that there was no significant loss of activity during sample transfers and that there was no significant self-absorption of activity from solids on the sample mount.

$\mathrm{NucFil}^{*}$ is a registered trademark of Nuclear Filter Technology, Incorporated, Golden, Colorado. The target practical quantification limits (PQLs) listed in the reference were met for all requested analyses except ruthenium/rhodium-106. The PQL for the total alpha analyses was on the order of $4 \times 10^{-7} \mathrm{uCi}$ per sample. This is equivalent to $0.4 \mathrm{pCi}$ per sample. The PQL for the total beta analyses was on the order of $3 \mathrm{pCi}$ per sample. The PQLs for the GEA analyses also varied slightly with the sample, and they varied with the radionuclide as well. All were less than $100 \mathrm{pCi}$ per sample for the requested radionuclides except for ruthenium/rhodium-106 which was approximately $150 \mathrm{pCi}$ per sample. 
Included in the total alpha and total beta results are the relative percent counting errors, which are a function of sample activity, sample size, background, and counting time. The counting error represents a $95 \%$ confidence limit about a sample result. For example, the total alpha result for sample 221T-99-032 was $4.25 \times 10^{-7} \mathrm{uCi}$ with a relative counting error of $117 \%$. Therefore, the true sample activity lies between $-7.22 \times 10^{-8}$ (less than background) and $9.22 \times 10^{-7} \mathrm{uCi}$ with a $95 \%$ certainty. Because these intervals are relatively large and the sample activities are very low, one should use caution when adding or subtracting results. Some samples with reported detectable amounts of activity may show no detectable amount if counted again for the same amount of time. The maximum counting error reported is 500 . A counting error of 500 indicates that the sample count was less than the background. 


\section{0-99-010}

\section{ATTACHMENT 1}

\section{Sample Breakdown Diagram}

Consisting of 2 pages,

Including cover page 


\section{Sample Breakdown and Analysis Scheme}
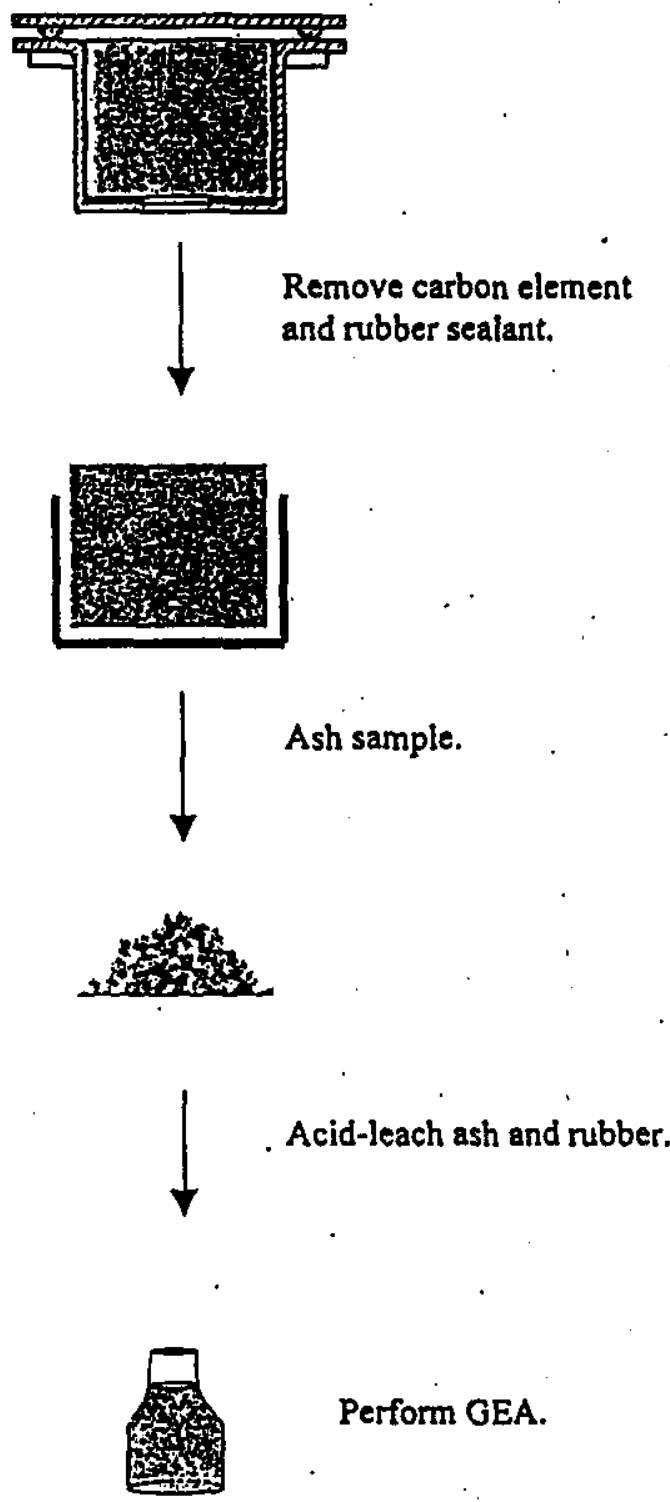

Perform GEA.

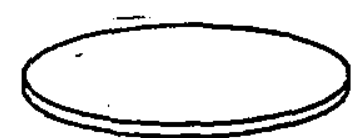

Mount liquid, dry, count for total alpha and total beta. 
31230-99-010

ATTACHMENT 2

\section{Analytical Results}

\section{Consisting of 24 pages,}

Including cover page 
Customer ID: 221T-99-025 Lab Sample \#: S99TP00051
Sample Date : 06/09/1999 11:10

Recv. Date : 05/28/1999 09:07

\begin{tabular}{|c|c|c|}
\hline PARAMETER & RESULTS & UNITS \\
\hline $\begin{array}{l}\text { Env AlphalBeta } \\
\text { Total Alpha (Each) }\end{array}$ & 3.63E-07 & $\mu \mathrm{Ci} /$ Sample \\
\hline Alpha Relative \% Counting Error & $1.33 E+02$ & $\%$ Ct. Error \\
\hline Total Beta (Each) & $1.44 E-05$ & $\mu \mathrm{Ci} /$ Sample \\
\hline Beta Relative \% Counting Error & $1.40 E+01$ & $\%$ Ct. Error \\
\hline $\begin{array}{l}\text { GEA Large Library Method } \\
\text { Beryllium-7 }\end{array}$ & $<7.207$ E-05 & $\mu \mathrm{Ci} /$ Sample \\
\hline Sodium-22 & $<9.973$ E-06 & $\mu \mathrm{Ci} /$ Sample \\
\hline Sodium-24 & $<9.949 E-06$ & $\mu \mathrm{Ci}$ Sample \\
\hline Potassium-40 & $<3.913 \mathrm{E}-04$ & $\mu \mathrm{Ci} / \mathrm{Sample}$ \\
\hline Argon-41 & $<1.535 \mathrm{E}-05$ & $\mu \mathrm{Ci} / \mathrm{Sample}$ \\
\hline Scandium-46 & $<1.467 \mathrm{E}-05$ & $\mu$ Ci/Sample \\
\hline Chromium-51 & $<6.484 E-05$ & $\mu \mathrm{Ci} / \mathrm{Sample}$ \\
\hline Manganese-54 & $<9.256 E-06$ & $\mu \mathrm{Ci} / \mathrm{Sample}$ \\
\hline Cobalt-56 & $<8.815 E-06$ & $\mu \mathrm{Ci} / \mathrm{Sample}$ \\
\hline Cobalt-57 & $<5.016 E-06$ & $\mu \mathrm{Ci} / \mathrm{Sample}$ \\
\hline Cobalt -58 & $<8.757 \mathrm{E}-06$ & $\mu \mathrm{Ci} / \mathrm{Sample}$ \\
\hline Iron-59 & $<1.775 E-05$ & $\mu \mathrm{Ci} /$ Sample \\
\hline Cobalt-60 & $<1.022 E-05$ & HCi/Sample \\
\hline Zinc-65 & $<2.057 E-05$ & $\mu \mathrm{Ci} / \mathrm{Sample}$ \\
\hline Selenium-75 & $<1.016 E-05$ & $\mu \mathrm{Ci} /$ Sample \\
\hline Krypton-85 & $<2.178 \mathrm{E}-03$ & $\mu \mathrm{Ci} / \mathrm{Sample}$ \\
\hline Strontium-85. & $<9.834 \mathrm{E}-06$ & $\mu$ Ci/Sample \\
\hline Yttrium-88 & $<7.617 \mathrm{E}-06$ & $\mu \mathrm{Ci} / \mathrm{Sample}$ \\
\hline Yttrium-91 & $<3.871 \mathrm{E}-03$ & $\mu \mathrm{Ci} /$ Sample \\
\hline Niobium-94 & $<8.510$ E-06 & $\mu \mathrm{Ci} / \mathrm{Sample}$ \\
\hline Zirconium/Niobium-95 & $<4.023 E-05$ & $\mu \mathrm{Ci} /$ Sample \\
\hline Ruthenium-103 & $<8.159 E-06$ & $\mu \mathrm{Ci} / \mathrm{Sample}$ \\
\hline Ruthenium/Rhodium-106 & $<1.594 \mathrm{E}-04$ & $\mu \mathrm{Ci} / \mathrm{Sample}$ \\
\hline Silver-108m & $<9.205 E-06$ & $\mu$ Ci/Sample \\
\hline Cadmium-109 & $<1.711 E-04$ & $\mu \mathrm{Ci} /$ Sample \\
\hline Silver-110m & $<8.750 \mathrm{E}-06$ & $\mu \mathrm{Ci} / \mathrm{Sample}$ \\
\hline Tin-113 & $<1.068 E-05$ & HCi/Sample \\
\hline Tellurium-123m & $<5.401 \mathrm{E}-06$ & $\mu \mathrm{Ci} / \mathrm{Sample}$ \\
\hline Antimony-124 & $<8.630 \mathrm{E}-06$ & $\mu \mathrm{Ci} /$ Sample \\
\hline Antimony-125 & $<2.275 E-05$ & $\mu \mathrm{Ci} / \mathrm{Sample}$ \\
\hline Tellurium-125m & $<1.625 E-03$ & $\mu \mathrm{Ci} /$ Sample \\
\hline lodine-129 & $<5.494 \mathrm{E}-03$ & $\mu \mathrm{Ci} /$ Sample \\
\hline lodine-131 & $<7.928 \mathrm{E}-06$ & $\mu \mathrm{Ci} /$ Sample \\
\hline Xenon-131m & $<2.339 \mathrm{E}-04$ & $\mu \mathrm{Ci} /$ Sample \\
\hline Barium-133 & $<1.043 E-05$ & $\mu \mathrm{Ci} /$ Sample \\
\hline Cesium-134 & $<8.461$ E-06 & 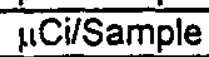 \\
\hline Cesium-136 & $<9.094 E-06$ & ${ }_{1} \mathrm{Ci} /$ Sample \\
\hline Cesium-137 & $<1.617$ E-05 & $\mu \mathrm{Ci} /$ Sample \\
\hline Cesium-138 & $<3.904 \mathrm{E}-05$ & $\mu \mathrm{Ci} / \mathrm{Sample}$ \\
\hline
\end{tabular}


Customer ID: 221 -99-025 (Continued) Lab Sample \#: S99TP00051

\begin{tabular}{|c|c|c|}
\hline Cerium-139 & $<5.648 E-06$ & $\mu \mathrm{Ci}$ Sample \\
\hline Barium-140 & $<3.227 E-05$ & HCi/Sample \\
\hline Lanthanum-140 & $<8.233 \mathrm{E}-06$ & $\mu \mathrm{Ci}$ Sample \\
\hline Cerium-141 & $<9.413 \mathrm{E}-06$ & $\mu \mathrm{Ci} / \mathrm{Sample}$ \\
\hline Cerium-144 & $<3.827 \mathrm{E}-05$ & $\mu \mathrm{Ci} /$ Sample \\
\hline Cerium/Praseodymium-144 & $<7.646 \mathrm{E}-05$ & $\mu \mathrm{Ci} /$ Sample \\
\hline Europium-152 & $<1.525 \mathrm{E}-05$ & $\mu \mathrm{Ci} /$ Sample \\
\hline Europium-154 & $<2.896 \mathrm{E}-05$ & $\mu \mathrm{Ci} /$ Sample \\
\hline Europium-155 & $<2.060 \mathrm{E}-05$ & $\mu \mathrm{Ci} /$ Sample \\
\hline Hafnium-181 & $<9.020$ E-06 & $\mu \mathrm{Ci} / \mathrm{Sample}$ \\
\hline Tantalum-182 & $<2.824 \mathrm{E}-05$ & $\mu \mathrm{Ci} / \mathrm{Sample}$ \\
\hline Mercury-203 & $<7.639$ E-06 & $\mu \mathrm{Ci} /$ Sample \\
\hline Bismuth-207 & $<8.102 E-06$ & $\mu$ Ci/Sample \\
\hline Thallium-208 & $<9.773 \mathrm{E}-05$ & $\mu \mathrm{Ci} / \mathrm{Sample}$ \\
\hline Lead-210 & $<2.367 \mathrm{E}-03$ & HCi/Sample \\
\hline Bismuth-212 & $<1.397 \mathrm{E}-04$ & $\mu \mathrm{Ci} /$ Sample \\
\hline Lead-212 & $<1.468 \mathrm{E}-05$ & $\mu \mathrm{Ci} /$ Sample \\
\hline Bismuth-214 & $<2.713 E-05$ & $\mu \mathrm{Ci} /$ Sample \\
\hline Lead-214 & $<9.012 \mathrm{E}-05$ & $\mu \mathrm{Ci} /$ Sample \\
\hline Radium-224 & $<1.549 \mathrm{E}-04$ & $\mu \mathrm{Ci} /$ Sample \\
\hline Radium-226 & $<1.476 \mathrm{E}-04$ & $\mu \mathrm{Ci} /$ Sample \\
\hline Actinium-228 & $<5.123 \mathrm{E}-05$ & HCi/Sample \\
\hline Thorium-228 & $<5.574 E-04$ & $\mu$ Ci/Sample \\
\hline Thorium-229 & $<2.464 \mathrm{E}-05$ & $\mu$ Ci/Sample \\
\hline Uranium-232 & $<1.160$ E-02 & „1Ci/Sample \\
\hline Protactinum-233 & $<1.642 \mathrm{E}-05$ & $\mu \mathrm{Ci} / \mathrm{S}$ ample \\
\hline Uranium/Thorium-233 & $<5.082 E-03$ & $\mu \mathrm{Ci} /$ Sample \\
\hline Protactinium-234m & $<1.515 \mathrm{E}-03$ & $\mu \mathrm{Ci} / \mathrm{S}$ ample \\
\hline Thorium-234 & $<3.968 \mathrm{E}-04$ & $\mu \mathrm{Ci} / \mathrm{S}$ ample \\
\hline Uranium-235 & $<9.005 \mathrm{E}-06$ & " Ci/Sample \\
\hline Neplunium-237 & $<5.465 \mathrm{E}-05$ & $\mu$ Ci/Sample \\
\hline Uranium-237 & $<1.877$ E-05 & $\mu$ Ci/Sample \\
\hline Neptunium-238 & $<3.350$ E-05 & $\mu \mathrm{Ci} /$ Sample \\
\hline Neptunium-239 & $<2.103 \mathrm{E}-05$ & $\mu \mathrm{Ci} /$ Sample \\
\hline Plutonium-239 & $<6.989 \mathrm{E}-02$ & $\mu$ Ci/Sample \\
\hline Americium-241 & $<5.575 \mathrm{E}-05$ & $\mu \mathrm{Ci} /$ Sample \\
\hline Americium-243 & $<1.648 E-05$ & $\mu \mathrm{Ci} / \mathrm{S} a m p l e$ \\
\hline
\end{tabular}


Customer ID: 221T-99-026 Lab Sample \#: S99TP00052
Sample Date : 06/09/1999 11:11

Recv. Date : 06/28/1999 09:07

\begin{tabular}{|c|c|c|}
\hline PARAMETER & RESULTS & UNITS \\
\hline $\begin{array}{l}\text { EnvAloha/Beta } \\
\text { Total Alpha (Each) }\end{array}$ & $<6.21 \mathrm{E}-07$ & uCi/Sample \\
\hline Alpha Relative \% Counting Error & $1.89 E+02$ & $\%$ C. Error \\
\hline Total Beta (Each) & $<1.80 \mathrm{E}-06$ & uCi/Sample \\
\hline Beta Relative \% Counting Error & $2.64 \mathrm{E}+02$ & $\%$ Ct. Error \\
\hline $\begin{array}{l}\text { GEA Large Library Method } \\
\text { Beryllium-7 }\end{array}$ & $<7.310 \mathrm{E}-05$ & $\mu \mathrm{Ci} /$ Sample \\
\hline Sodium-22 & $<9.839 \mathrm{E}-06$ & $\mu \mathrm{Ci} / \mathrm{Sample}$ \\
\hline Sodium-24 & $<9.125 E-06$ & $\mu \mathrm{Ci} / \mathrm{Sample}$ \\
\hline Potassium-40 & $<3.927 E-04$ & $\mu \mathrm{Ci} / \mathrm{Sample}$ \\
\hline Argon-41 & $<1.450 E-05$ & $\mu \mathrm{Ci} / \mathrm{Sample}$ \\
\hline Scandium-46 & $<1.313 E-05$ & $\mu \mathrm{Ci} / \mathrm{S}$ ample \\
\hline Chromium-51 & $<6.359 \mathrm{E}-05$ & $1 \mathrm{Ci} / \mathrm{Sample}$ \\
\hline Manganese-54 & $<9.715 \mathrm{E}-06$ & $\mu \mathrm{Ci} / \mathrm{Sample}$ \\
\hline Cobalt-56 & $<8.954 \mathrm{E}-06$ & $\mu \mathrm{Ci} / \mathrm{Sample}$ \\
\hline Cobalt-57 & $<5.112 \mathrm{E}-06$ & HCi/Sample \\
\hline Cobalt-58 & $<8.545 E-06$ & $\mu \mathrm{Ci} /$ Sample \\
\hline rron-59 & $<1.831 \mathrm{E}-05$ & $\mu \mathrm{Ci} / \mathrm{Sample}$ \\
\hline Cobalt-60 & $<1.011 \mathrm{E}-05$ & $\mathrm{CC}$ i/Sample \\
\hline Zinc-65 & $<2.180 \mathrm{E}-05$ & $\mu \mathrm{Ci} / \mathrm{Sample}$ \\
\hline Selenium-75 & $<1.016 \mathrm{E}-05$ & $\mu \mathrm{Ci} /$ Sample \\
\hline Krypton-85 & $<2.128 E-03$ & $\mu \mathrm{Ci} / \mathrm{Sample}$ \\
\hline Strontium-85 & $<9.604 \mathrm{E}-06$ & $\mu \mathrm{Ci} / \mathrm{Sample}$ \\
\hline Yttrium-88 & $<7.679 E-06$ & $\mu \mathrm{Ci} / \mathrm{S}$ ample \\
\hline Yttrium-91 & $<3.822 E-03$ & $\mu \mathrm{Ci} / \mathrm{Sample}$ \\
\hline Niobium-94 & $<9.202 E-06$ & $\mu \mathrm{Ci} / \mathrm{Sample}$ \\
\hline Zirconium/Niobium-95 & $<3.865 \mathrm{E}-05$ & HCi/Sample \\
\hline Ruthenium-103 & $<8.369 E-06$ & $\mu \mathrm{Ci} / \mathrm{Sample}$ \\
\hline Ruthenium/Rhodium-106 & $<1.588 \mathrm{E}-04$ & $\mu \mathrm{Ci} / \mathrm{S}$ ample \\
\hline Silver-108m & $<9.205 E-06$ & $\mu \mathrm{Ci} /$ Sample \\
\hline Cadmium-109 & $<1.683 E-04$ & $\mu \mathrm{Ci} / \mathrm{Sample}$ \\
\hline Silver-110m & $<8.202 E-06$ & $\mu \mathrm{Ci} /$ Sample \\
\hline Tin-113 & $<1.084 \mathrm{E}-05$ & $\mu \mathrm{Ci} / \mathrm{Sample}$ \\
\hline Tellurium-123m & $<5.364 \mathrm{E}-06$ & $\mu \mathrm{Ci} / \mathrm{Sample}$ \\
\hline Antimony-124 & $<8.043 E-06$ & $\mu \mathrm{Ci} /$ Sample \\
\hline Antimony-125 & $<2.446 E-05$ & $\mu \mathrm{Ci} /$ Sample \\
\hline Tellurium-125m & $<1.619 \mathrm{E}-03$ & $\mu \mathrm{Ci} / \mathrm{Sample}$ \\
\hline lodine-129 & $<5.711 \mathrm{E}-03$ & \\
\hline Sodine-131 & $<8.354 E-06$ & $\mu \mathrm{Ci} /$ Sample \\
\hline Xenon-131m & $<2.420 \mathrm{E}-04$ & $\mu \mathrm{Ci} / \mathrm{Sample}$ \\
\hline Barium-133 & $<1.067$ E-05 & $\mu \mathrm{Ci} /$ Sample \\
\hline Cesium-134 & $<7.896 \mathrm{E}-06$ & $\mu \mathrm{Ci} /$ Sample \\
\hline Cesium-136 & $<8.716$ E-06 & $\mu \mathrm{Ci} /$ Sample \\
\hline Cesium-137 & $<1.447 \mathrm{E}-05$ & $\mu \mathrm{Ci} /$ Sample \\
\hline Cesium-138 & $<4.036 \mathrm{E}-05$ & $\mu$ Ci/Sample \\
\hline
\end{tabular}


Customer 1D: 221T-99-026 (Continued) Lab Sample \#: S99TP00052

\begin{tabular}{|l|c|c|}
\hline Cerium-139 & $<5.814 \mathrm{E}-06$ & $\mu \mathrm{Ci} /$ Sample \\
\hline Barium-140 & $<3.028 \mathrm{E}-05$ & $\mu \mathrm{Ci} /$ Sample \\
\hline Lanthanum-140 & $<9.040 \mathrm{E}-06$ & $\mu \mathrm{Ci} /$ Sample \\
\hline Cerium-141 & $<9.392 \mathrm{E}-06$ & $\mu \mathrm{Ci} /$ Sample \\
\hline Cerium-144 & $<3.945 \mathrm{E}-05$ & $\mu \mathrm{Ci} /$ Sample \\
\hline Cerium/Praseodymium-144 & $<7.882 \mathrm{E}-05$ & $\mu \mathrm{Ci} /$ Sample \\
\hline Europium-152 & $<1.547 \mathrm{E}-05$ & $\mu \mathrm{Ci} /$ Sample \\
\hline Europium-154 & $<2.858 \mathrm{E}-05$ & $\mu \mathrm{Ci} /$ Sample \\
\hline Europium-155 & $<1.984 \mathrm{E}-05$ & $\mu \mathrm{Ci} /$ Sample \\
\hline Hafnium-181 & $<9.405 \mathrm{E}-06$ & $\mu \mathrm{Ci} /$ Sample \\
\hline Tantalum-182 & $<2.854 \mathrm{E}-05$ & $\mu \mathrm{Ci} /$ Sample \\
\hline Mercury-203 & $<7.513 \mathrm{E}-06$ & $\mu \mathrm{Ci} /$ Sample \\
\hline Bismuth-207 & $<7.958 \mathrm{E}-06$ & $\mu \mathrm{Ci} /$ Sample \\
\hline Thallium-208 & $<9.632 \mathrm{E}-05$ & $\mu \mathrm{Ci} /$ Sample \\
\hline Lead-210 & $<2.311 \mathrm{E}-03$ & $\mu \mathrm{Ci} /$ Sample \\
\hline Bismuth-212 & $<1.403 \mathrm{E}-04$ & $\mu \mathrm{Ci} /$ Sample \\
\hline Lead-212 & $<1.491 \mathrm{E}-05$ & $\mu \mathrm{Ci} /$ Sample \\
\hline Bismuth-214 & $<2.728 \mathrm{E}-05$ & $\mu \mathrm{Ci} /$ Sample \\
\hline Lead-214 & $<9.188 \mathrm{E}-05$ & $\mu \mathrm{Ci} /$ Sample \\
\hline Radium-224 & $<1.517 \mathrm{E}-04$ & $\mu \mathrm{Ci} /$ Sample \\
\hline Radium-226 & $<1.440 \mathrm{E}-04$ & $\mu \mathrm{Ci} /$ Sample \\
\hline Actinium-228 & $<4.953 \mathrm{E}-05$ & $\mu \mathrm{Ci} /$ Sample \\
\hline Thorium-228 & $<5.565 \mathrm{E}-04$ & $\mu \mathrm{Ci} /$ Sample \\
\hline Thorium-229 & $<2.418 \mathrm{E}-05$ & $\mu \mathrm{Ci} /$ Sample \\
\hline Uranium-232 & $<1.133 \mathrm{E}-02$ & $\mu \mathrm{Ci} /$ Sample \\
\hline Protactinum-233 & $<1.664 \mathrm{E}-05$ & $\mu \mathrm{Ci} /$ Sample \\
\hline Uranium/Thorium-233 & $<5.340 \mathrm{E}-03$ & $\mu \mathrm{Ci} /$ Sample \\
\hline Protactinium-234m & $<1.591 \mathrm{E}-03$ & $\mu \mathrm{Ci} /$ Sample \\
\hline Thorium-234 & $<3.995 \mathrm{E}-04$ & $\mu \mathrm{Ci} /$ Sample \\
\hline Uranium-235 & $<8.840 \mathrm{E}-06$ & $\mu \mathrm{Ci} /$ Sample \\
\hline Neptunium-237 & $<5.275 \mathrm{E}-05$ & $\mu \mathrm{Ci} /$ Sample \\
\hline Uranium-237 & $<1.920 \mathrm{E}-05$ & $\mu \mathrm{Ci} /$ Sample \\
\hline Neptunium-238 & $<3.503 \mathrm{E}-05$ & $\mu \mathrm{Ci} /$ Sample \\
\hline Neptunium-239 & $<2.070 \mathrm{E}-05$ & $\mu \mathrm{Ci} /$ Sample \\
\hline Plutonium-239 & $<7.025 \mathrm{E}-02$ & $\mu \mathrm{Ci} /$ Sample \\
\hline Americium-241 & $<5.453 \mathrm{E}-05$ & $\mu \mathrm{Ci} /$ Sample \\
\hline Americium-243 & $<1.591 \mathrm{E}-05$ & $\mu \mathrm{Ci} /$ Sample \\
\hline
\end{tabular}


Customer 1D: 221T-99-027 Lab Sample \#: S99TP00053
Sample Date : 06/09/1999 11:13

Recv. Date : 06/28/1999 09:07

\begin{tabular}{|c|c|c|}
\hline PARAMETER & RESULTS & UNITS \\
\hline $\begin{array}{l}\text { Env Alpha/Bela } \\
\text { Total Alpha (Each) }\end{array}$ & $<5.46 \mathrm{E}-07$ & uCi/Sample \\
\hline Alpha Relative \% Counting Error & $2.45 \mathrm{E}+02$ & $\%$ Ct. Error \\
\hline Total Beta (Each) & $1.85 \mathrm{E}-06$ & uCi/Sample \\
\hline Beta Relative \% Counting Error & $8.29 E+01$ & \% Ct. Error \\
\hline $\begin{array}{l}\text { GEA Laroe Library Method } \\
\text { Beryllium-7 }\end{array}$ & $<7.115 E-05$ & $\mu \mathrm{Ci} /$ Sample \\
\hline Sodium-22 & $<9.728 \mathrm{E}-06$ & Ci/Sample \\
\hline Sodium-24 & $<8.519 E-06$ & $\mu \mathrm{Ci} /$ Sample \\
\hline Potassium -40 & $<3.849 \mathrm{E}-04$ & \\
\hline Argon-41 & $<1.542 \mathrm{E}-05$ & $\mu \mathrm{Ci} /$ Sample \\
\hline Scandium-46 & $<1.381 \mathrm{E}-05$ & $\mu$ Ci/Sample \\
\hline Chromium-51 & $<6.366 \mathrm{E}-05$ & "Cilsample \\
\hline Manganese-54 & $<9.796 \mathrm{E}-06$ & $\mu \mathrm{Ci} / \mathrm{S}$ ample \\
\hline Cobalt-56 & $<8.928 E-06$ & $\mu \mathrm{Ci} /$ Sample \\
\hline Cobalt-57 & $<5.107 \mathrm{E}-06$ & $\mu$ Ci/Sample \\
\hline Cobait-58 & $<8.606 \mathrm{E}-06$ & HCi/Sample \\
\hline Iron-59 & $<1.872 \mathrm{E}-05$ & $\mu$ Ci/Sample \\
\hline Cobalt-60 & $<1.006 \mathrm{E}-05$ & $\mu$ CilSample \\
\hline Zinc-65 & $<2.052 E-05$ & $\mu \mathrm{Ci} / \mathrm{Sample}$ \\
\hline Selenium-75 & $<1.035 E-05$ & $\mu \mathrm{Ci} /$ Sample \\
\hline Krypton-85 & $<2.102 \mathrm{E}-03$ & $\mu \mathrm{Ci} /$ Sample \\
\hline Strontium-85 & $<9.494 E-06$ & $\mu \mathrm{Ci} /$ Sample \\
\hline Yttrium-88 & $<7.168 \mathrm{E}-06$ & $\mu \mathrm{Ci} /$ Sample \\
\hline Yttrium-91 & $<3.867 \mathrm{E}-03$ & $\mu \mathrm{Ci} /$ Sample \\
\hline Niobium-94 & $<8.636 \mathrm{E}-06$ & $\mu \mathrm{Ci} /$ Sample \\
\hline Zirconium/Niobium-95 & $<3.863 E-05$ & $\mu \mathrm{Ci} / \mathrm{Sample}$ \\
\hline Ruthenium-103 & $<8.611 E-06$ & $\mu$ CirSample \\
\hline Ruthenium/Rhodium-106 & $<1.590 E-04$ & $\mu \mathrm{Ci} /$ Sample \\
\hline Silver-108m & $<9.122 \mathrm{E}-06$ & $\mu \mathrm{Ci} /$ Sample \\
\hline Cadmium-109 & $<1.676 E-04$ & $\mu \mathrm{Ci} /$ Sample \\
\hline Silver-110m & $<8.530$ E-06 & $\mu \mathrm{Ci} /$ Sample \\
\hline Tin-113 & $<1.067 \mathrm{E}-05$ & $\mu \mathrm{Ci} / \mathrm{Sample}$ \\
\hline Tellurium-123m & $<5.490 \mathrm{E}-06$ & $\mu \mathrm{Ci}$ Sample \\
\hline Antimony-124 & $<8.061 E-06$ & $\mu \mathrm{Ci} /$ Sample \\
\hline Antimony-125 & $<2.336 E-05$ & $\mu$ Cirsample \\
\hline Tellurium-125m & $<1.624 E-03$ & $\mu \mathrm{Ci} /$ Sample \\
\hline Iodine-129 & $<5.612 \mathrm{E}-03$ & $\mu \mathrm{Ci} /$ Sample \\
\hline lodine-131 & $<8.147 E-06$ & $\mu \mathrm{Ci} / \mathrm{Sample}$ \\
\hline Xenon-131m & $<2.378 E-04$ & $\mu \mathrm{Ci} /$ Sample \\
\hline Barivim-133 & $<1.060$ E-05 & $\mu \mathrm{Ci} /$ Sample \\
\hline Cesium-134 & $<8.286 E-06$ & $\mu \mathrm{Ci} /$ Sample \\
\hline Cesium-136 & $<8.647 E-06$ & $\mu \mathrm{Ci}$ Sample \\
\hline Cesium-137 & $<1.482 E-05$ & $\mu \mathrm{Ci}$ Sample \\
\hline Cesium-138 & $<3.890$ E-05 & $\mu \mathrm{Ci} /$ Sample \\
\hline
\end{tabular}


Customer ID: 221T-99-027 (Continued)

Lab Sample \#: S99TP00053

\begin{tabular}{|l|c|c|}
\hline Cerium-139 & $<5.834 \mathrm{E}-06$ & $\mu \mathrm{Ci} /$ Sample \\
\hline Barium-140 & $<3.210 \mathrm{E}-05$ & $\mu \mathrm{Ci} /$ Sample \\
\hline Lanthanum-140 & $<8.389 \mathrm{E}-06$ & $\mu \mathrm{Ci} /$ Sample \\
\hline Cerium-141 & $<9.268 \mathrm{E}-06$ & $\mu \mathrm{Ci} /$ Sample \\
\hline Cerium-144 & $<3.849 \mathrm{E}-05$ & $\mu \mathrm{Ci} /$ Sample \\
\hline Cerium/Praseodymium-144 & $<7.691 \mathrm{E}-05$ & $\mu \mathrm{Ci} /$ Sample \\
\hline Europium-152 & $<1.543 \mathrm{E}-05$ & $\mu \mathrm{Ci} /$ Sample \\
\hline Europium-154 & $<2.827 \mathrm{E}-05$ & $\mu \mathrm{Ci} /$ Sample \\
\hline Europium-155 & $<1.937 \mathrm{E}-05$ & $\mu \mathrm{Ci} /$ Sample \\
\hline Hafnium-181 & $<9.527 \mathrm{E}-06$ & $\mu \mathrm{Ci} /$ Sample \\
\hline Tantalum-182 & $<2.770 \mathrm{E}-05$ & $\mu \mathrm{Ci} /$ Sample \\
\hline Mercury-203 & $<7.327 \mathrm{E}-06$ & $\mu \mathrm{Ci} /$ Sample \\
\hline Bismuth-207 & $<8.078 \mathrm{E}-06$ & $\mu \mathrm{Ci} /$ Sample \\
\hline Thallium-208 & $<9.299 \mathrm{E}-05$ & $\mu \mathrm{Ci} /$ Sample \\
\hline Lead-210 & $<2.289 \mathrm{E}-03$ & $\mu \mathrm{Ci} /$ Sample \\
\hline Bismuth-212 & $<1.395 \mathrm{E}-04$ & $\mu \mathrm{Ci} /$ Sample \\
\hline Lead-212 & $<1.502 \mathrm{E}-05$ & $\mu \mathrm{Ci} /$ Sample \\
\hline Bismuth-214 & $<2.662 \mathrm{E}-05$ & $\mu \mathrm{Ci} /$ Sample \\
\hline Lead-214 & $<8.863 \mathrm{E}-05$ & $\mu \mathrm{Ci} /$ Sample \\
\hline Radium-224 & $<1.544 \mathrm{E}-04$ & $\mu \mathrm{Ci} /$ Sample \\
\hline Radium-226 & $<1.472 \mathrm{E}-04$ & $\mu \mathrm{Ci} /$ Sample \\
\hline Actinium-228 & $<4.818 \mathrm{E}-05$ & $\mu \mathrm{Ci} /$ Sample \\
\hline Thorium-228 & $<5.660 \mathrm{E}-04$ & $\mu \mathrm{Ci} /$ Sample \\
\hline Thorium-229 & $<2.420 \mathrm{E}-05$ & $\mu \mathrm{Ci} /$ Sample \\
\hline Uranium-232 & $<1.163 \mathrm{E}-02$ & $\mu \mathrm{Ci} /$ Sample \\
\hline Protactinum-233 & $<1.616 \mathrm{E}-05$ & $\mu \mathrm{Ci} /$ Sample \\
\hline Uranium Thorium-233 & $<5.148 \mathrm{E}-03$ & $\mu \mathrm{Ci} /$ Sample \\
\hline Protactinium-234m & $<1.633 \mathrm{E}-03$ & $\mu \mathrm{Ci} /$ Sample \\
\hline Thorium-234 & $<3.934 \mathrm{E}-04$ & $\mu \mathrm{Ci} /$ Sample \\
\hline Uranium-235 & $<8.986 \mathrm{E}-06$ & $\mu \mathrm{Ci} /$ Sample \\
\hline Neptunium-237 & $<5.149 \mathrm{E}-05$ & $\mu \mathrm{Ci} /$ Sample \\
\hline Uranium-237 & $<1.937 \mathrm{E}-05$ & $\mu \mathrm{Ci} /$ Sample \\
\hline Neptunium-238 & $<3.342 \mathrm{E}-05$ & $\mu \mathrm{Ci} /$ Sample \\
\hline Neptunium-239 & $<2.015 \mathrm{E}-05$ & $\mu \mathrm{Ci} /$ Sample \\
\hline Plutonium-239 & $<6.885 \mathrm{E}-02$ & $\mu \mathrm{Ci} /$ Sample \\
\hline Americium-241 & $<5.642 \mathrm{E}-05$ & $\mu \mathrm{Ci} /$ Sample \\
\hline Americium-243 & $<1.635 \mathrm{E}-05$ & $\mu \mathrm{Ci} /$ Sample \\
\hline
\end{tabular}


Customer ID: 221T-99-028

Lab Sample \#: S99TP00054

Sample Date : 06/09/1999 11:15

Recv. Date : 06/28/1999 09:07

\begin{tabular}{|c|c|c|}
\hline PARAMETER & RESULTS & UNITS \\
\hline $\begin{array}{l}\text { Env Alpha/Beta } \\
\text { Total Alpha (Each) }\end{array}$ & $<6.21 \mathrm{E}-07$ & uCi/Sample \\
\hline Alpha Relative \% Counting Error & $1.89 E+02$ & $\%$ Ct. Error \\
\hline Total Beta (Each) & $1.78 E-06$ & UCi/Sample \\
\hline Beta Relative \% Counting Error & $8.60 E+01$ & $\%$ ct. Error \\
\hline $\begin{array}{l}\text { GEA Large Library Melhod } \\
\text { Beryllium-7 }\end{array}$ & $<7.253 \mathrm{E}-05$ & $\mu \mathrm{Ci} / \mathrm{Sample}$ \\
\hline Sodium-22 & $<9.829 E-06$ & HCi/Sample \\
\hline Sodium-24 & $<9.368 \mathrm{E}-06$ & HCi/Sample \\
\hline Polassium-40 & $<3.796 \mathrm{E}-04$ & $\mu \mathrm{Ci} / \mathrm{S}$ ample \\
\hline Argon-41 & $<1.547 \mathrm{E}-05$ & $\mu \mathrm{Ci} /$ Sample \\
\hline Scandium-46 & $<1.337 E-05$ & $\mu \mathrm{Ci} / \mathrm{Sample}$ \\
\hline Chromium-51 & $<6.120$ E-05 & $\mu \mathrm{Ci} /$ Sample \\
\hline Manganese-54 & $<9.578 E-06$ & $\mu \mathrm{Ci} /$ Sample \\
\hline Cobalt-56 & $<9.072 E-06$ & HCi/Sample \\
\hline Cobalt-57 & $<5.092 \mathrm{E}-06$ & $\mu \mathrm{Ci} / \mathrm{Sample}$ \\
\hline Cobalt-58 & $<8.375 \mathrm{E}-06$ & $\mu \mathrm{Ci} /$ Sample \\
\hline Iron-59 & $<1.780 \mathrm{E}-05$ & $\mu \mathrm{Ci} /$ Sample \\
\hline Cobalt- 60 & $<9.104 \mathrm{E}-06$ & $\mu \mathrm{Ci} /$ Sample \\
\hline Zinc-65 & $<2.107 \mathrm{E}-05$ & $\mu \mathrm{Ci} /$ Sample \\
\hline Selenium-75 & $<1.039 E-05$ & \\
\hline Krypton-85 & $<2.113 E-03$ & HCi/Sample \\
\hline Strontium-85 & $<9.537$ E-06 & $\mu \mathrm{Ci} / \mathrm{Sample}$ \\
\hline Yttrium-88 & $<8.774 \mathrm{E}-06$ & $\mu \mathrm{Ci} /$ Sample \\
\hline Yttrium-91 & $<3.690 E-03$ & $\mu \mathrm{Ci} /$ Sample \\
\hline Niobium-94 & $<8.595 E-06$ & $\mu \mathrm{Ci} / \mathrm{Sample}$ \\
\hline Zirconium/Niobium-95 & $<3.871 E-05$ & $\mu \mathrm{Ci} / \mathrm{Sample}$ \\
\hline Ruthenium-103 & $<8.049 E-06$ & $\mu \mathrm{Ci} /$ Sample \\
\hline Ruthenium/Rhodium-106 & $<1.641 E-04$ & HCi/Sample \\
\hline Silver-108m & $<9.017 \mathrm{E}-06$ & HCi/Sample \\
\hline Cadmium-109 & $<1.673 \mathrm{E}-04$ & $\mu \mathrm{Ci} /$ Sample \\
\hline Silver-110m & $<9.014 \mathrm{E}-06$ & $\mu \mathrm{Ci} /$ Sample \\
\hline Tin-113 & $<1.062 E-05$ & $\mu \mathrm{Ci} /$ Sample \\
\hline Tellurium-123m & $<5.410 \mathrm{E}-06$ & „LCi/Sample \\
\hline Antimony-124 & $<8.218 E-06$ & $\mu \mathrm{Ci} /$ Sample \\
\hline Antimony-125 & $<2.450$ E-05 & $\mu \mathrm{Ci} /$ Sample \\
\hline Tellurium-125m & $<1.631 E-03$ & $\mu \mathrm{Ci} /$ Sample \\
\hline lodine-129 & $<5.544 E-03$ & HCi/Sample \\
\hline lodine-131 & $<8.247 E-06$ & $\mu$ CilSample \\
\hline Xenon-131m & $<2.387$ E.04 & $\mu \mathrm{Ci} /$ Sample \\
\hline Barium-133 & $<1.083 E-05$ & $\mu \mathrm{Ci} /$ Sample \\
\hline Cesium-134 & $<8.411 E-06$ & $\mu \mathrm{Ci} /$ Sample \\
\hline Cesium-136 & $<8.457 \mathrm{E}-06$ & $\mu \mathrm{Ci} / \mathrm{Sample}$ \\
\hline Cesium-137 & $<1.469$ E-05 & „Ci/Sample \\
\hline Cesium-138 & $<3.966 E-05$ & $\mu \mathrm{Ci} /$ Sample \\
\hline
\end{tabular}


Customer ID: 221T-99-028 (Continued) Lab Sample \#: S99TP00054

\begin{tabular}{|l|c|c|}
\hline Cerium-139 & $<5.932 \mathrm{E}-06$ & $\mu \mathrm{Ci} /$ Sample \\
\hline Barium-140 & $<3.070 \mathrm{E}-05$ & $\mu \mathrm{Ci} /$ Sample \\
\hline Lanthanum-140 & $<8.780 \mathrm{E}-06$ & $\mu \mathrm{Ci} /$ Sample \\
\hline Cerium-141 & $<9.287 \mathrm{E}-06$ & $\mu \mathrm{Ci} /$ Sample \\
\hline Cerium-144 & $<3.970 \mathrm{E}-05$ & $\mu \mathrm{Ci} /$ Sample \\
\hline Cerium/Praseodymium-144 & $<7.933 \mathrm{E}-05$ & $\mu \mathrm{Ci} /$ Sample \\
\hline Europium-152 & $<1.545 \mathrm{E}-05$ & $\mu \mathrm{Ci} /$ Sample \\
\hline Europium-154 & $<2.858 \mathrm{E}-05$ & $\mu \mathrm{Ci} /$ Sample \\
\hline Europium-155 & $<1.980 \mathrm{E}-05$ & $\mu \mathrm{Ci} /$ Sample \\
\hline Hafnium-181 & $<9.321 \mathrm{E}-06$ & $\mu \mathrm{Ci} /$ Sample \\
\hline Tantalum-182 & $<2.799 \mathrm{E}-05$ & $\mu \mathrm{Ci} /$ Sample \\
\hline Mercury-203 & $<7.414 \mathrm{E}-06$ & $\mu \mathrm{Ci} /$ Sample \\
\hline Bismuth-207 & $<8.057 \mathrm{E}-06$ & $\mu \mathrm{Ci} /$ Sample \\
\hline Thallium-208 & $<9.671 \mathrm{E}-05$ & $\mu \mathrm{Ci} /$ Sample \\
\hline Lead-210 & $<2.225 \mathrm{E}-03$ & $\mu \mathrm{Ci} /$ Sample \\
\hline Bismuth-212 & $<1.457 \mathrm{E}-04$ & $\mu \mathrm{Ci} /$ Sample \\
\hline Lead-212 & $<1.469 \mathrm{E}-05$ & $\mu \mathrm{Ci} /$ Sample \\
\hline Bismuth-214 & $<2.660 \mathrm{E}-05$ & $\mu \mathrm{Ci} /$ Sample \\
\hline Lead-214 & $<8.730 \mathrm{E}-05$ & $\mu \mathrm{Ci} /$ Sample \\
\hline Radium-224 & $<1.503 \mathrm{E}-04$ & $\mu \mathrm{Ci} /$ Sample \\
\hline Radium-226 & $<1.458 \mathrm{E}-04$ & $\mu \mathrm{Ci} /$ Sample \\
\hline Actinium-228 & $<5.046 \mathrm{E}-05$ & $\mu \mathrm{Ci} /$ Sample \\
\hline Thorium-228 & $<5.624 \mathrm{E}-04$ & $\mu \mathrm{Ci} /$ Sample \\
\hline Thorium-229 & $<2.409 \mathrm{E}-05$ & $\mu \mathrm{Ci} /$ Sample \\
\hline Uranium-232 & $<1.117 \mathrm{E}-02$ & $\mu \mathrm{Ci} /$ Sample \\
\hline Protactinum-233 & $<1.617 \mathrm{E}-05$ & $\mu \mathrm{Ci} /$ Sample \\
\hline Uranium/Thorium-233 & $<5.255 \mathrm{E}-03$ & $\mu \mathrm{Ci} /$ Sample \\
\hline Protactinium-234m & $<1.456 \mathrm{E}-03$ & $\mu \mathrm{Ci} /$ Sample \\
\hline Thorium-234 & $<4.035 \mathrm{E}-04$ & $\mu \mathrm{Ci} /$ Sample \\
\hline Uranium-235 & $<8.855 \mathrm{E}-06$ & $\mu \mathrm{Ci} /$ Sample \\
\hline Neptunium-237 & $<5.258 \mathrm{E}-05$ & $\mu \mathrm{Ci} /$ Sample \\
\hline Uranium-237 & $<1.919 \mathrm{E}-05$ & $\mu \mathrm{Ci} /$ Sample \\
\hline Neptunium-238 & $<3.362 \mathrm{E}-05$ & $\mu \mathrm{Ci} /$ Sample \\
\hline Neptunium-239 & $<2.050 \mathrm{E}-05$ & $\mu \mathrm{Ci} /$ Sample \\
\hline Plutonium-239 & $<6.890 \mathrm{E}-02$ & $\mu \mathrm{Ci} /$ Sample \\
\hline Americium-241 & $<5.643 \mathrm{E}-05$ & $\mu \mathrm{Ci} /$ Sample \\
\hline Americium-243 & $<1.517 \mathrm{E}-05$ & $\mu \mathrm{Ci} /$ Sample \\
\hline
\end{tabular}




\begin{tabular}{|c|c|c|}
\hline PARAMETER & RESULTS & UNITS \\
\hline $\begin{array}{l}\text { Env AlohalBela } \\
\text { Total Alpha (Each) }\end{array}$ & $<3.24 \mathrm{E}-07$ & uCi/Sample \\
\hline Alpha Relative \% Counting Error & $5.00 E+02$ & $\%$ C. Error \\
\hline Total Beta (Each) & $3.36 E-06$ & uCi/Sample \\
\hline Beta Relative \% Counting Error & $4.77 E+01$ & $\%$ Ct. Error \\
\hline $\begin{array}{l}\text { GEA Large Librax Method } \\
\text { Beryllium-7 }\end{array}$ & $<6.815 E-05$ & $\mu \mathrm{Ci} /$ Sample \\
\hline Sodium-22 & $<9.986 \mathrm{E}-06$ & $\mu \mathrm{Ci} /$ Sample \\
\hline Sodium-24 & $<9.327 E-06$ & $\mu \mathrm{Ci} /$ Sample \\
\hline Potassium-40 & $<3.810 \mathrm{E}-04$ & HCi/Sample \\
\hline Argon-41 & $<1.584 E-05$ & $\mu \mathrm{Ci} /$ Sample \\
\hline Scandium-46 & $<1.340$ E-05 & $\mu \mathrm{Ci} /$ Sample \\
\hline Chromium-51 & $<6.355 E-05$ & $\mu \mathrm{Ci} /$ Sample \\
\hline Manganese-54 & $<8.830 E-06$ & $\mu \mathrm{Ci} /$ Sample \\
\hline Cobalt-56 & $<8.804 E-06$ & $\mu \mathrm{Ci} /$ Sample \\
\hline Cobalt-57 & $<5,065 \mathrm{E}-06$ & $\mu \mathrm{Ci} /$ Sample \\
\hline Cobalt-58 & $<8.831 E-06$ & $\mu \mathrm{Ci} /$ Sample \\
\hline iron-59 & $<1.777$ E-05 & $\mu \mathrm{Ci} / \mathrm{Sample}$ \\
\hline Cobalt-60 & $<9.971 \mathrm{E}-06$ & $\mu \mathrm{Ci} /$ Sample \\
\hline Zinc-65 & $<1.971 \mathrm{E}-05$ & $\mu \mathrm{Ci} / \mathrm{Sample}$ \\
\hline Selenium-75 & $<1.001$ E-05 & $\mu \mathrm{Ci} /$ Sample \\
\hline Krypton-85 & $<2.081 E-03$ & $\mathrm{Ci}$ Cisample \\
\hline Strontium-85 & $<9.393 \mathrm{E}-06$ & $\mu \mathrm{Ci} /$ Sample \\
\hline Yttrium-88 & $<7.585 E-06$ & $\mu \mathrm{Ci} /$ Sample \\
\hline Yttrium-91 & $<3.714 \mathrm{E}-03$ & $\mu \mathrm{Ci} / \mathrm{Sample}$ \\
\hline Niobium-94 & $<8.859 E-06$ & $\mu \mathrm{Ci} /$ Sample \\
\hline Zirconium/Niobium-95 & $<3.769 E-05$ & 스/Sample \\
\hline Ruthenium-103 & $<8.251 E-06$ & " Ci/Sample \\
\hline Ruthenium/Rhodium-106 & $<1.578 \mathrm{E}-04$ & $\mu \mathrm{Ci} / \mathrm{Sample}$ \\
\hline Silver-108m & $<8.898 E-06$ & "Ci/Sample \\
\hline Cadmium-109 & $<1.671 \mathrm{E}-04$ & $\mu \mathrm{Ci} /$ Sample \\
\hline Silver-110m & $<8.831 E-06$ & $\mu \mathrm{Ci} / \mathrm{Sample}$ \\
\hline Tin-113 & $<1.093 E-05$ & $\mu \mathrm{Ci} /$ Sample \\
\hline Tellurium-123m & $<5.336 \mathrm{E}-06$ & \\
\hline Antimony-124 & $<8.269 \mathrm{E}-06$ & $\mu \mathrm{Ci} /$ Sample \\
\hline Antimony-125 & $<2.363 E-05$ & $\mu \mathrm{Ci} / \mathrm{Sample}$ \\
\hline Tellurium-125m & $<1.593 \mathrm{E}-03$ & بCi/Sample \\
\hline Lodine-129 & $<5.601 \mathrm{E}-03$ & $\mu \mathrm{Ci} /$ Sample \\
\hline lodine-131 & $<8.075 E-06$ & $\mu \mathrm{Ci} / \mathrm{Sample}$ \\
\hline Xenon-131m & $<2.315 \mathrm{E}-04$ & $\mu \mathrm{Ci} / \mathrm{S}$ ample \\
\hline Barium-133 & $<1.069 E-05$ & $\mu \mathrm{Ci} /$ Sample \\
\hline Cesium-134 & $<8.084 E-06$ & "Ci/Sample \\
\hline Cesium-136 & $<8.744 E-06$ & $\mu \mathrm{Ci} /$ Sample \\
\hline Cesium-137 & $<1.457$ E-05 & $\mu \mathrm{Ci} / \mathrm{Sample}$ \\
\hline Cesium-138 & $<3.903 E-05$ & $\mu \mathrm{Ci} /$ Sample \\
\hline
\end{tabular}


Customer ID: 221T-99-029 (Continued) Lab Sample \#: S99TP00055

\begin{tabular}{|l|c|c|}
\hline Cerium-139 & $<5.630 \mathrm{E}-06$ & $\mu \mathrm{Ci} /$ Sample \\
\hline Barium-140 & $<3.208 \mathrm{E}-05$ & $\mu \mathrm{Ci} /$ Sample \\
\hline Lanthanum-140 & $<8.497 \mathrm{E}-06$ & $\mu \mathrm{Ci} /$ Sample \\
\hline Cerium-141 & $<9.015 \mathrm{E}-06$ & $\mu \mathrm{Ci} /$ Sample \\
\hline Cerium-144 & $<3.923 \mathrm{E}-05$ & $\mu \mathrm{Ci} /$ Sample \\
\hline Cerium/Praseodymium-144 & $<7.838 \mathrm{E}-05$ & $\mu \mathrm{Ci} /$ Sample \\
\hline Europium-152 & $<1.522 \mathrm{E}-05$ & $\mu \mathrm{Ci} /$ Sample \\
\hline Europium-154 & $<2.900 \mathrm{E}-05$ & $\mu \mathrm{Ci} /$ Sample \\
\hline Europium-155 & $<1.984 \mathrm{E}-05$ & $\mu \mathrm{Ci} /$ Sample \\
\hline Hafnium-181 & $<8.915 \mathrm{E}-06$ & $\mu \mathrm{Ci} /$ Sample \\
\hline Tantalum-182 & $<2.805 \mathrm{E}-05$ & $\mu \mathrm{Ci} /$ Sample \\
\hline Mercury-203 & $<7.554 \mathrm{E}-06$ & $\mu \mathrm{Ci} /$ Sample \\
\hline Bismuth-207 & $<7.820 \mathrm{E}-06$ & $\mu \mathrm{Ci} /$ Sample \\
\hline Thallium-208 & $<9.693 \mathrm{E}-05$ & $\mu \mathrm{Ci} /$ Sample \\
\hline Lead-210 & $<2.304 \mathrm{E}-03$ & $\mu \mathrm{Ci} /$ Sample \\
\hline Bismuth-212 & $<1.431 \mathrm{E}-04$ & $\mu \mathrm{Ci} /$ Sample \\
\hline Lead-212 & $<1.454 \mathrm{E}-05$ & $\mu \mathrm{Ci} /$ Sample \\
\hline Bismuth-214 & $<2.610 \mathrm{E}-05$ & $\mu \mathrm{Ci} /$ Sample \\
\hline Lead-214 & $<8.818 \mathrm{E}-05$ & $\mu \mathrm{Ci} /$ Sample \\
\hline Radium-224 & $<1.540 \mathrm{E}-04$ & $\mu \mathrm{Ci} /$ Sample \\
\hline Radium-226 & $<1.496 \mathrm{E}-04$ & $\mu \mathrm{Ci} /$ Sample \\
\hline Actinium-228 & $<5.151 \mathrm{E}-05$ & $\mu \mathrm{Ci} /$ Sample \\
\hline Thorium-228 & $<5.504 \mathrm{E}-04$ & $\mu \mathrm{Ci} /$ Sample \\
\hline Thorium-229 & $<2.418 \mathrm{E}-05$ & $\mu \mathrm{Ci} /$ Sample \\
\hline Uranium-232 & $<1.136 \mathrm{E}-02$ & $\mu \mathrm{Ci} /$ Sample \\
\hline Protactinum-233 & $<1.640 \mathrm{E}-05$ & $\mu \mathrm{Ci} /$ Sample \\
\hline Uranium $/$ Thorium-233 & $<5.168 \mathrm{E}-03$ & $\mu \mathrm{Ci} /$ Sample \\
\hline Protactinium-234m & $<1.451 \mathrm{E}-03$ & $\mu \mathrm{Ci} /$ Sample \\
\hline Thorium-234 & $<4.033 \mathrm{E}-04$ & $\mu \mathrm{Ci} /$ Sample \\
\hline Uranium-235 & $<8.999 \mathrm{E}-06$ & $\mu \mathrm{Ci} /$ Sample \\
\hline Neptunium-237 & $<5.277 \mathrm{E}-05$ & $\mu \mathrm{Ci} /$ Sample \\
\hline Uranium-237 & $<1.834 \mathrm{E}-05$ & $\mu \mathrm{Ci} /$ Sample \\
\hline Neptunium-238 & $<3.615 \mathrm{E}-05$ & $\mu \mathrm{Ci} /$ Sample \\
\hline Neptunium-239 & $<2.014 \mathrm{E}-05$ & $\mu \mathrm{Ci} /$ Sample \\
\hline Plutonium-239 & $<6.698 \mathrm{E}-02$ & $\mu \mathrm{Ci} /$ Sample \\
\hline Americium-241 & $<5.529 \mathrm{E}-05$ & $\mu \mathrm{Ci} /$ Sample \\
\hline Americium-243 & $<1.614 \mathrm{E}-05$ & $\mu \mathrm{Ci} /$ Sample \\
\hline
\end{tabular}


Customer ID: 221T-99-030 Lab Sample \#: S99TP00056
Sample Date : 06/10/1999 11:15 Recv. Date : 06/28/1999 09:07

\begin{tabular}{|c|c|c|}
\hline PARAMETER & RESULTS & UNITS \\
\hline $\begin{array}{l}\text { Env Alpha/Bela } \\
\text { Total Alpha (Each) }\end{array}$ & $<5.46 \mathrm{E}-07$ & |uCi/Sample \\
\hline Alpha Relative \% Counting Error & $2.45 E+02$ & $\%$ Ct. Error \\
\hline Total Beta (Each) & $3.59 E-05$ & uCi/Sample \\
\hline Beta Relative \% Counting Error & $7.33 \bar{E}+00$ & $\%$ Ct. Error \\
\hline $\begin{array}{l}\text { GEA Large Libran Method } \\
\text { Beryllium-7 }\end{array}$ & $<7.177$ E-05 & $\mu \mathrm{Ci} /$ Sample \\
\hline Sodium-22 & $\because<1.026 E-05$ & $\mu \mathrm{Ci} / \mathrm{S}$ ample \\
\hline Sodium-24 & $<9.197 \mathrm{E}-06$ & $\mu \mathrm{Ci} / \mathrm{Sample}$ \\
\hline Potassium-40 & $<3.921 \mathrm{E}-04$ & $\mu \mathrm{Ci} /$ Sample \\
\hline Argon-41 & $<1.458 \mathrm{E}-05$ & HCi/Sample \\
\hline Scandium-46 & $<1.370 \mathrm{E}-05$ & $\mu$ Cirsample \\
\hline Chromium-51 & $<6.484 E-05$ & $\mu$ Ci/Sample \\
\hline Manganese-54 & $<8.980 \mathrm{E}-06$ & $\mu \mathrm{Ci} / \mathrm{Sample}$ \\
\hline Cobalt-56 & $<9.167$ E-06 & $\mu \mathrm{Ci} /$ Sample \\
\hline Cobalt-57 & $<5.031 E-06$ & HCi/Sample \\
\hline Cobalt-58 & $<8.894 \mathrm{E} .06$ & HCi/Sample \\
\hline Iron-59 & $<1.837 \mathrm{E}-05$ & $\mu \mathrm{Ci} / \mathrm{Sample}$ \\
\hline Cobalt-60 & $<9.923 E-06$ & $\mu \mathrm{Ci} /$ Sample \\
\hline Zinc-65 & $<2.046 \mathrm{E}-05$ & $\mu \mathrm{Ci} / \mathrm{Sample}$ \\
\hline Selenium-75 & $<9.859 \mathrm{E}-06$ & $\mu \mathrm{Ci} / \mathrm{Sample}$ \\
\hline Krypton-85 & $<2.116 \mathrm{E}-03$ & $\mu$ Ci/Sample \\
\hline Strontium-85 & $<9.549 E-06$ & $\mu \mathrm{Ci} /$ Sample \\
\hline Yttrium-88 & $<8.118 E-06$ & $\mu \mathrm{Ci} / \mathrm{Sample}$ \\
\hline Yttrium-91 & $<3.916 \mathrm{E}-03$ & $\mu \mathrm{Ci} / \mathrm{Sample}$ \\
\hline Niobium-94 & $<9.527 E-06$ & $\mu \mathrm{Ci} /$ Sample \\
\hline Zirconium/Niobium-95 & $<3.586 E-05$ & $\mu \mathrm{Ci} / \mathrm{Sample}$ \\
\hline Ruthenium-103 & $<8.275 \mathrm{E}-06$ & $\mu \mathrm{Ci} /$ Sample \\
\hline Ruthenium/Rhodium-106 & $<1.609 \mathrm{E}-04$ & $\mu \mathrm{Ci} / \mathrm{Sample}$ \\
\hline Silver-108m & $<8.853 \mathrm{E}-06$ & $\mu \mathrm{Ci} / \mathrm{Sample}$ \\
\hline Cadmium-109 & $<1.699 E-04$ & $\mu \mathrm{Ci} /$ Sample \\
\hline Silver-110m & $<8.333 E-06$ & $\mu \mathrm{Ci} / \mathrm{Sample}$ \\
\hline Tin-113 & $<1.081 E-05$ & $\mu \mathrm{Ci} /$ Sample \\
\hline Tellurium-123m & $<5.327 E-06$ & $\mu \mathrm{Ci} /$ Sample \\
\hline Antimony-124 & $<7.837$ E-06 & $\mu \mathrm{Ci} / \mathrm{Sample}$ \\
\hline Antimony-125 & $<2.411 E-05$ & $\mu \mathrm{Ci} /$ Sample \\
\hline Tellurium-125m & $<1.652 \mathrm{E}-03$ & $\mu \mathrm{Ci} / \mathrm{Sample}$ \\
\hline lodine-129 & $<5.364 \mathrm{E}-03$ & $\mu \mathrm{Ci} /$ Sample \\
\hline lodine-131 & $<8.224 E-06$ & $\mu \mathrm{Ci} /$ Sample \\
\hline Xenon-131m & $<2.349$ E-04 & $\mu \mathrm{Ci} / \mathrm{Sample}$ \\
\hline Barium-133 & $<1.062 E-05$ & MCi/Sample \\
\hline Cesium-134 & $<7.874 E-06$ & $\mu \mathrm{Ci} /$ Sample \\
\hline Cesium-136 & $<8.863 E-06$ & $\mu \mathrm{Ci} /$ Sample \\
\hline Cesium-137 & $<3.957$ E-05 & $\mu \mathrm{Ci} /$ Sample \\
\hline Cesium-138 & $<5.723 E-06$ & $\mu \mathrm{Ci} /$ Sample \\
\hline
\end{tabular}


Customer ID: 221T-99-030 (Continued)

Lab Sample \#: S99TP00056

\begin{tabular}{|l|c|c|}
\hline Cerium-139 & $<3.096 \mathrm{E}-05$ & $\mu \mathrm{Ci} /$ Sample \\
\hline Barium-140 & $<8.631 \mathrm{E}-06$ & $\mu \mathrm{Ci} /$ Sample \\
\hline Lanthanum-140 & $<9.241 \mathrm{E}-06$ & $\mu \mathrm{Ci} /$ Sample \\
\hline Cerium-141 & $<3.885 \mathrm{E}-05$ & $\mu \mathrm{Ci} /$ Sample \\
\hline Cerium-144 & $<7.764 \mathrm{E}-05$ & $\mu \mathrm{Ci} /$ Sample \\
\hline Cerium/Praseodymium-144 & $<1.529 \mathrm{E}-05$ & $\mu \mathrm{Ci} /$ Sample \\
\hline Europium-152 & $<2.978 \mathrm{E}-05$ & $\mu \mathrm{Ci} /$ Sample \\
\hline Europium-154 & $<1.977 \mathrm{E}-05$ & $\mu \mathrm{Ci} /$ Sample \\
\hline Europium-155 & $<9.250 \mathrm{E}-06$ & $\mu \mathrm{Ci} /$ Sample \\
\hline Hafnium-181 & $<2.772 \mathrm{E}-05$ & $\mu \mathrm{Ci} /$ Sample \\
\hline Tantalum-182 & $<7.531 \mathrm{E}-06$ & $\mu \mathrm{Ci} /$ Sample \\
\hline Mercury-203 & $<7.578 \mathrm{E}-06$ & $\mu \mathrm{Ci} /$ Sample \\
\hline Bismuth-207 & $<9.609 \mathrm{E}-05$ & $\mu \mathrm{Ci} /$ Sample \\
\hline Thallium-208 & $<9.609 \mathrm{E}-05$ & $\mu \mathrm{Ci} /$ Sample \\
\hline Lead-210 & $<2.252 \mathrm{E}-03$ & $\mu \mathrm{Ci} /$ Sample \\
\hline Bismuth-212 & $<1.347 \mathrm{E}-04$ & $\mu \mathrm{Ci} /$ Sample \\
\hline Lead-212 & $<1.500 \mathrm{E}-05$ & $\mu \mathrm{Ci} /$ Sample \\
\hline Bismuth-214 & $<2.554 \mathrm{E}-05$ & $\mu \mathrm{Ci} /$ Sample \\
\hline Lead-214 & $<8.914 \mathrm{E}-05$ & $\mu \mathrm{Ci} /$ Sample \\
\hline Radium-224 & $<1.538 \mathrm{E}-04$ & $\mu \mathrm{Ci} /$ Sample \\
\hline Radium-226 & $<1.448 \mathrm{E}-04$ & $\mu \mathrm{Ci} /$ Sample \\
\hline Actinium-228 & $<4.943 \mathrm{E}-05$ & $\mu \mathrm{Ci} /$ Sample \\
\hline Thorium-228 & $<5.452 \mathrm{E}-04$ & $\mu \mathrm{Ci} /$ Sample \\
\hline Thorium-229 & $<2.475 \mathrm{E}-05$ & $\mu \mathrm{Ci} /$ Sample \\
\hline Uranium-232 & $<1.122 \mathrm{E}-02$ & $\mu \mathrm{Ci} /$ Sample \\
\hline Protactinum-233 & $<1.623 \mathrm{E}-05$ & $\mu \mathrm{Ci} /$ Sample \\
\hline Uranium/Thorium-233 & $<5.428 \mathrm{E}-03$ & $\mu \mathrm{Ci} /$ Sample \\
\hline Protactinium-234m & $<1.568 \mathrm{E}-03$ & $\mu \mathrm{Ci} /$ Sample \\
\hline Thorium-234 & $<4.028 \mathrm{E}-04$ & $\mu \mathrm{Ci} /$ Sample \\
\hline Uranium-235 & $<8.813 \mathrm{E}-06$ & $\mu \mathrm{Ci} /$ Sample \\
\hline Neptunium-237 & $<5.262 \mathrm{E}-05$ & $\mu \mathrm{Ci} /$ Sample \\
\hline Uranium-237 & $<1.927 \mathrm{E}-05$ & $\mu \mathrm{Ci} /$ Sample \\
\hline Neptunium-238 & $<3.561 \mathrm{E}-05$ & $\mu \mathrm{Ci} /$ Sample \\
\hline Neptunium-239 & $<6.804 \mathrm{E}-02$ & $\mu \mathrm{Ci} /$ Sample \\
\hline Plutonium-239 & $<5.501 \mathrm{E}-05$ & $\mu \mathrm{Ci} /$ Sample \\
\hline Americium-241 & $\mu \mathrm{Ci} /$ Sample \\
\hline Americium-243 & & \\
\hline
\end{tabular}


Customer ID: 221T-99-031

Lab Sample \#: S99TP00057
Sample Date : 06/10/1999 13:10

Recv. Date : 06/28/1999 09:07

\begin{tabular}{|c|c|c|}
\hline PARAMETER & RESULTS & UNITS \\
\hline $\begin{array}{l}\text { Env AlphalBeta } \\
\text { Total Alpha (Each) }\end{array}$ & $<3.24 \mathrm{E}-07$ & uCi/Sample \\
\hline Alpha Relative \% Counting Error & $5.00 E+02$ & $\%$ Ct. Error \\
\hline Total Beta (Each) & 1.89E-06 & UCi/Sample \\
\hline Beta Relative \% Counting Error & $8.15 E+01$ & $\%$ Ct. Error \\
\hline $\begin{array}{l}\text { GEA Large Library Methed } \\
\text { Beryllium-7 }\end{array}$ & $<7.148 E-05$ & $\mu \mathrm{Ci} /$ Sample \\
\hline Sodium-22 & $<1.022 \mathrm{E}-05$ & $\mu \mathrm{Ci} / \mathrm{Sample}$ \\
\hline Sodium-24 & $<8.795 E-06$ & $\mu \mathrm{Ci} /$ Sample \\
\hline Potassium -40 & $<3.868 \mathrm{E}-04$ & $\mu \mathrm{Ci} /$ Sample \\
\hline Argon-41 & $<1.649 E-05$ & $\mu \mathrm{Ci} /$ Sample \\
\hline Scandium-46 & $<1.361$ E-05 & $\mu \mathrm{Ci} / \mathrm{Sample}$ \\
\hline Chromium-51 & $<6.573 E-05$ & $\mu$ Ci/Sample \\
\hline Manganese-54 & $<9.155 E-06$ & $\mu \mathrm{Ci} / \mathrm{Sample}$ \\
\hline Cobalt-56 & $<8.751 \mathrm{E}-06$ & $\mu \mathrm{Ci} /$ Sample \\
\hline Cobalt-57 & $<5.143 E-06$ & $\mu \mathrm{Ci} / \mathrm{Sample}$ \\
\hline Cobalt-58 & $<8.987$ E-06 & $\mu \mathrm{Ci} /$ Sample \\
\hline Iron-59 & $<1.781$ E-05 & $\mu \mathrm{Ci}$ Sample \\
\hline Cobalt-60 & $<1.011 \mathrm{E}-05$ & $\mu \mathrm{Ci} /$ Sample \\
\hline Zinc-65 & $<2.067 \mathrm{E}-05$ & $\mu \mathrm{Ci} /$ Sample \\
\hline Selenium-75 & $<9.995 E-06$ & $\mu \mathrm{Ci} / \mathrm{Sample}$ \\
\hline Krypton-85 & $<2.108 E-03$ & $\mu \mathrm{Ci} /$ Sample \\
\hline Strontium-85 & $<9.509 E-06$ & $\mu \mathrm{Ci} /$ Sample \\
\hline Yttrium-88 & $<7.233 \mathrm{E}-06$ & $\mu \mathrm{Ci} / \mathrm{Sample}$ \\
\hline Yttrium-91 & $<3.693 \mathrm{E}-03$ & $\mu \mathrm{Ci} / \mathrm{Sample}$ \\
\hline Niobium-94 & $<8.892 \mathrm{E}-06$ & $\mu \mathrm{Ci} / \mathrm{Sample}$ \\
\hline Zirconium/Niobium-95 & $<3.880$ E-05 & $\mu \mathrm{Ci} /$ Sample \\
\hline Ruthenium-103 & $<8.086 \mathrm{E}-06$ & $\mu \mathrm{Ci} /$ Sample \\
\hline Ruthenium/Rhodium-106 & $<1.567 \mathrm{E}-04$ & $\mu \mathrm{Ci} /$ Sample \\
\hline Silver-108m & $<9.356 E-06$ & $\mu \mathrm{Ci} /$ Sample \\
\hline Cadmium-109 & $<1.639 \mathrm{E}-04$ & $\mu \mathrm{Ci} /$ Sample \\
\hline Silver-110m & $<8.490 \mathrm{E}-06$ & $\mu \mathrm{Ci} /$ Sample \\
\hline $\operatorname{Tin}-113$ & $<1.045 E-05$ & $\mu \mathrm{Ci} / \mathrm{Sample}$ \\
\hline Tellurium-123m & $<5.263 \mathrm{E}-06$ & $\mu \mathrm{Ci} /$ Sample \\
\hline Antimony-124 & $<8.304$ E-06 & $\mu \mathrm{Ci} /$ Sample \\
\hline Antimony-125 & $<2.389 E-05$ & $\mu \mathrm{Ci} /$ Sample \\
\hline Tellurium-125m & $<1.633 E-03$ & $\mu \mathrm{Ci} /$ Sample \\
\hline lodine-129 & $<5.434 \mathrm{E}-03$ & $\mu \mathrm{Ci} /$ Sample \\
\hline lodine-131 & $<8.219 \mathrm{E}-06$ & $\mu \mathrm{Ci} /$ Sample \\
\hline Xenon-131m & $<2.252 E-04$ & $\mu \mathrm{Ci} /$ Sample \\
\hline Barium-133 & $<1.079 \mathrm{E}-05$ & $\mu \mathrm{Ci} /$ Sample \\
\hline Cesium-134 & $<7.903 E-06$ & $\mu \mathrm{Ci} /$ Sample \\
\hline Cesium-136 & $<8.875 E-06$ & $\mu \mathrm{Ci} /$ Sample \\
\hline Cesium-137 & $<1.431 E-05$ & $\mu \mathrm{Ci} / \mathrm{Sample}$ \\
\hline Cesium-138 & $<4.293 E-05$ & HCi/Sample \\
\hline
\end{tabular}


Customer ID: 221T-99-031 (Continued)

Lab Sample \#: S99TP00057

\begin{tabular}{|l|c|c|}
\hline Cerium-139 & $<5.482 \mathrm{E}-06$ & $\mu \mathrm{Ci} /$ Sample \\
\hline Barium-140 & $<3.150 \mathrm{E}-05$ & $\mu \mathrm{Ci} /$ Sample \\
\hline Lanthanum-140 & $<7.542 \mathrm{E}-06$ & $\mu \mathrm{Ci} /$ Sample \\
\hline Cerium-141 & $<9.042 \mathrm{E}-06$ & $\mu \mathrm{Ci} /$ Sample \\
\hline Cerium-144 & $<3.880 \mathrm{E}-05$ & $\mu \mathrm{Ci} /$ Sample \\
\hline Cerium/Praseodymium-144 & $<7.753 \mathrm{E}-05$ & $\mu \mathrm{Ci} /$ Sample \\
\hline Europium-152 & $<1.565 \mathrm{E}-05$ & $\mu \mathrm{Ci} /$ Sample \\
\hline Europium-154 & $<2.970 \mathrm{E}-05$ & $\mu \mathrm{Ci} /$ Sample \\
\hline Europium-155 & $<1.963 \mathrm{E}-05$ & $\mu \mathrm{Ci} /$ Sample \\
\hline Hafnium-181 & $<9.201 \mathrm{E}-06$ & $\mu \mathrm{Ci} /$ Sample \\
\hline Tantalum-182 & $<2.761 \mathrm{E}-05$ & $\mu \mathrm{Ci} /$ Sample \\
\hline Mercury-203 & $<7.595 \mathrm{E}-06$ & $\mu \mathrm{Ci} /$ Sample \\
\hline Bismuth-207 & $<8.228 \mathrm{E}-06$ & $\mu \mathrm{Ci} /$ Sample \\
\hline Thallium-208 & $<9.773 \mathrm{E}-05$ & $\mu \mathrm{Ci} /$ Sample \\
\hline Lead-210 & $<2.261 \mathrm{E}-03$ & $\mu \mathrm{Ci} /$ Sample \\
\hline Bismuth-212 & $<1.383 \mathrm{E}-04$ & $\mu \mathrm{Ci} /$ Sample \\
\hline Lead-212 & $<1.450 \mathrm{E}-05$ & $\mu \mathrm{Ci} /$ Sample \\
\hline Bismuth-214 & $<2.593 \mathrm{E}-05$ & $\mu \mathrm{Ci} /$ Sample \\
\hline Lead-214 & $<8.682 \mathrm{E}-05$ & $\mu \mathrm{Ci} /$ Sample \\
\hline Radium-224 & $<1.512 \mathrm{E}-04$ & $\mu \mathrm{Ci} /$ Sample \\
\hline Radium-226 & $<1.441 \mathrm{E}-04$ & $\mu \mathrm{Ci} /$ Sample \\
\hline Actinium-228 & $<4.942 \mathrm{E}-05$ & $\mu \mathrm{Ci} /$ Sample \\
\hline Thorium-228 & $<5.484 \mathrm{E}-04$ & $\mu \mathrm{Ci} /$ Sample \\
\hline Thorium-229 & $<2.364 \mathrm{E}-05$ & $\mu \mathrm{Ci} /$ Sample \\
\hline Uranium-232 & $<1.131 \mathrm{E}-02$ & $\mu \mathrm{Ci} /$ Sample \\
\hline Protactinum-233 & $<1.586 \mathrm{E}-05$ & $\mu \mathrm{Ci} /$ Sample \\
\hline Uranium/Thorium-233 & $<5.189 \mathrm{E}-03$ & $\mu \mathrm{Ci} /$ Sample \\
\hline Protactinium-234m & $<1.496 \mathrm{E}-03$ & $\mu \mathrm{Ci} /$ Sample \\
\hline Thorium-234 & $<3.992 \mathrm{E}-04$ & $\mu \mathrm{Ci} /$ Sample \\
\hline Uranium-235 & $<8.743 \mathrm{E}-06$ & $\mu \mathrm{Ci} /$ Sample \\
\hline Neptunium-237 & $<5.228 \mathrm{E}-05$ & $\mu \mathrm{Ci} /$ Sample \\
\hline Uranium-237 & $<1.867 \mathrm{E}-05$ & $\mu \mathrm{Ci} /$ Sample \\
\hline Neptunium-238 & $<3.494 \mathrm{E}-05$ & $\mu \mathrm{Ci} /$ Sample \\
\hline Neptunium-239 & $<1.999 \mathrm{E}-05$ & $\mu \mathrm{Ci} /$ Sample \\
\hline Plutonium-239 & $<6.662 \mathrm{E}-02$ & $\mu \mathrm{Ci} /$ Sample \\
\hline Americium-241 & $<5.485 \mathrm{E}-05$ & $\mu \mathrm{Ci} /$ Sample \\
\hline Americium-243 & $\mu 1.606 \mathrm{E}-05$ & $\mu \mathrm{Ci} /$ Sample \\
\hline
\end{tabular}


Customer 1D: 221T-99-032 Lab Sample \#: S99TP00058
Sample Date : 06/10/1999 14:40

Recv. Date : 06/28/1999 09:07

\begin{tabular}{|c|c|c|}
\hline PARAMETER & RESULTS & UNITS \\
\hline $\begin{array}{l}\text { EnvAlpha/Beta } \\
\text { Total Aipha (Each) }\end{array}$ & $4.25 \mathrm{E}-07$ & UCi/Sample \\
\hline Alpha Relative \% Counting Error & $1.17 E+02$ & $\%$ Ct. Error \\
\hline Total Beta (Each) & 9.79E-06 & uCi/Sample \\
\hline Beta Relative \% Counting Error & $1.89 E+01$ & $\%$ Ct. Error \\
\hline $\begin{array}{l}\text { GEA Large Library Method } \\
\text { Beryllium-7 }\end{array}$ & $<6.946 \mathrm{E}-05$ & $\mu \mathrm{Ci} /$ Sample \\
\hline Sodium-22 & $<1.071$ E-05 & $\mu \mathrm{Ci} / \mathrm{Sample}$ \\
\hline Sodium-24 & $<9.425 E-06$ & $\mu \mathrm{Ci} /$ Sample \\
\hline Potassium-40 & $<3.860 E-04$ & $\mu \mathrm{Ci} / \mathrm{Sample}$ \\
\hline Argon-41 & $<1.488 \mathrm{E}-05$ & $\mu \mathrm{Ci} /$ Sample \\
\hline Scandium-46 & $<1.392 E-05$ & $\mu \mathrm{Ci} /$ Sample \\
\hline Chromium-51 & $<6.325 E-05$ & $\mu \mathrm{Ci} / \mathrm{S} a m p l e$ \\
\hline Manganese-54 & $<9.286 E-06$ & $\mu \mathrm{Ci} / \mathrm{Sample}$ \\
\hline Cobalt-56 & $<9.100$ E-06. & $\mu \mathrm{Ci} /$ Sample \\
\hline Cobalt-57 & $<4.971 \mathrm{E}-06$ & $\mu \mathrm{Ci} / \mathrm{S}$ ample \\
\hline Cobalt-58 & $<8.316 E-06$ & $\mu \mathrm{Ci} / \mathrm{Sample}$ \\
\hline Iron-59 & $<1.940 \mathrm{E}-05$ & $\mu \mathrm{Ci} / \mathrm{Sample}$ \\
\hline Cobalt-60 & $<9.670 E-06$ & $\mu \mathrm{Ci} /$ Sample \\
\hline Zinc-65 & $<2.014 \mathrm{E}-05$ & $\mu \mathrm{Ci} / \mathrm{Sample}$ \\
\hline Selenium-75 & $<9.851 \mathrm{E}-06$ & $\mu \mathrm{Ci} / \mathrm{Sample}$ \\
\hline Krypton-85 & $<2.120$ E-03 & HCi/Sample \\
\hline Strontium-85 & $<9.569 \mathrm{E}-06$ & $\mu \mathrm{Ci} / \mathrm{S}$ ample \\
\hline Yttrium-88 & $<8.459 E-06$ & $\mu \mathrm{Ci} / \mathrm{Sample}$ \\
\hline Yttrium-91 & $<3.586 \mathrm{E}-03$ & $\mu \mathrm{Ci} /$ Sample \\
\hline Niobium-94 & $<8.643 E-06$ & $\mu \mathrm{Ci} / \mathrm{Sample}$ \\
\hline Zirconium/Niobium-95 & $<3.801 E-05$ & $\mu \mathrm{Ci} / \mathrm{Sample}$ \\
\hline Ruthenium-103 & $<7.908 E-06$ & $\mu \mathrm{Ci} / \mathrm{Sample}$ \\
\hline Ruthenium/Rhodium-106 & $<1.624 E-04$ & $\mu \mathrm{Ci} / \mathrm{S} a m p l e$ \\
\hline Silver-108m & $<9.520$ E-06 & HCi/Sample \\
\hline Cadmium-109 & $<1.656 \mathrm{E}-04$ & $\mu \mathrm{Ci} /$ Sample \\
\hline Silver-110m & $<8.757 E-06$ & $\mu \mathrm{Ci} / \mathrm{Sample}$ \\
\hline Tin-113 & $<1.065 \mathrm{E}-05$ & $\mu \mathrm{Ci} / \mathrm{Sample}$ \\
\hline Tellurium-123m & $<5.358 \mathrm{E}-06$ & $\mu \mathrm{Ci} / \mathrm{Sample}$ \\
\hline Antimony-124 & $<8.296 E-06$ & $\mu \mathrm{Ci} / \mathrm{Sample}$ \\
\hline Antimony-125 & $<2.351 E-05$ & $\mu \mathrm{Ci} / \mathrm{Sample}$ \\
\hline Tellurium-125m & $<1.595 E .03$ & $\mu \mathrm{Ci} / \mathrm{Sample}$ \\
\hline lodine-129 & $<5.402 E-03$ & $\mu$ Ci/Sample \\
\hline lodine-131 & $<8.243 E-06$ & $\mu \mathrm{Ci} / \mathrm{Sample}$ \\
\hline Xenon-131m & $<2.338 \mathrm{E}-04$ & $\mu \mathrm{Ci} / \mathrm{Sample}$ \\
\hline Barium-133 & $<1.057 \mathrm{E}-05$ & $\mu \mathrm{Ci} / \mathrm{S}$ ample \\
\hline Cesium-134 & $<8.358 E-06$ & $\mu \mathrm{Ci} /$ Sample \\
\hline Cesium-136 & $<8.252 E-06$ & HCi/Sample \\
\hline Cesium-137 & $<1.476 \mathrm{E}-05$ & $\mu \mathrm{Ci} / \mathrm{Sample}$ \\
\hline Cesium-138 & $<3.793$ E-05 & $\mu \mathrm{Ci} / \mathrm{Sample}$ \\
\hline
\end{tabular}


Customer ID: 221T-99-032 (Continued) Lab Sample \#: S99TP00058

\begin{tabular}{|l|c|c|}
\hline Cerium-139 & $<5.642 \mathrm{E}-06$ & $\mu \mathrm{Ci} /$ Sample \\
\hline Barium-140 & $<3.197 \mathrm{E}-05$ & $\mu \mathrm{Ci} /$ Sample \\
\hline Lanthanum-140 & $<8.730 \mathrm{E}-06$ & $\mu \mathrm{Ci} /$ Sample \\
\hline Cerium-141 & $<9.405 \mathrm{E}-06$ & $\mu \mathrm{Ci} /$ Sample \\
\hline Cerium-144 & $<3.891 \mathrm{E}-05$ & $\mu \mathrm{Ci} /$ Sample \\
\hline Cerium/Praseodymium-144 & $<7.774 \mathrm{E}-05$ & $\mu \mathrm{Ci} /$ Sample \\
\hline Europium-152 & $<1.505 \mathrm{E}-05$ & $\mu \mathrm{Ci} /$ Sample \\
\hline Europium-154 & $<3.113 \mathrm{E}-05$ & $\mu \mathrm{Ci} /$ Sample \\
\hline Europium-155 & $<1.963 \mathrm{E}-05$ & $\mu \mathrm{Ci} /$ Sample \\
\hline Hafnium-181 & $<9.385 \mathrm{E}-06$ & $\mu \mathrm{Ci} /$ Sample \\
\hline Tantalum-182 & $<2.840 \mathrm{E}-05$ & $\mu \mathrm{Ci} /$ Sample \\
\hline Mercury-203 & $<7.301 \mathrm{E}-06$ & $\mu \mathrm{Ci} /$ Sample \\
\hline Bismuth-207 & $<8.281 \mathrm{E}-06$ & $\mu \mathrm{Ci} /$ Sample \\
\hline Thallium-208 & $<9.707 \mathrm{E}-05$ & $\mu \mathrm{Ci} /$ Sample \\
\hline Lead-210 & $<2.326 \mathrm{E}-03$ & $\mu \mathrm{Ci} /$ Sample \\
\hline Bismuth-212 & $<1.402 \mathrm{E}-04$ & $\mu \mathrm{Ci} /$ Sample \\
\hline Lead-212 & $<1.477 \mathrm{E}-05$ & $\mu \mathrm{Ci} /$ Sample \\
\hline Bismuth-214 & $<2.662 \mathrm{E}-05$ & $\mu \mathrm{Ci} /$ Sample \\
\hline Lead-214 & $<8.688 \mathrm{E}-05$ & $\mu \mathrm{Ci} /$ Sample \\
\hline Radium-224 & $<1.519 \mathrm{E}-04$ & $\mu \mathrm{Ci} /$ Sample \\
\hline Radium-226 & $<1.482 \mathrm{E}-04$ & $\mu \mathrm{Ci} /$ Sample \\
\hline Actinium-228 & $<5.162 \mathrm{E}-05$ & $\mu \mathrm{Ci} /$ Sample \\
\hline Thorium-228 & $<5.449 \mathrm{E}-04$ & $\mu \mathrm{Ci} /$ Sample \\
\hline Thorium-229 & $<2.372 \mathrm{E}-05$ & $\mu \mathrm{Ci} /$ Sample \\
\hline Uranium-232 & $<1.141 \mathrm{E}-02$ & $\mu \mathrm{Ci} /$ Sample \\
\hline Protactinum-233 & $<1.659 \mathrm{E}-05$ & $\mu \mathrm{Ci} /$ Sample \\
\hline Uranium/Thorium-233 & $<5.294 \mathrm{E}-03$ & $\mu \mathrm{Ci} /$ Sample \\
\hline Protactinium-234m & $<1.526 \mathrm{E}-03$ & $\mu \mathrm{Ci} /$ Sample \\
\hline Thorium-234 & $<3.977 \mathrm{E}-04$ & $\mu \mathrm{Ci} /$ Sample \\
\hline Uranium-235 & $<9.040 \mathrm{E}-06$ & $\mu \mathrm{Ci} /$ Sample \\
\hline Neptunium-237 & $<5.223 \mathrm{E}-05$ & $\mu \mathrm{Ci} /$ Sample \\
\hline Uranium-237 & $<1.840 \mathrm{E}-05$. & $\mu \mathrm{Ci} /$ Sample \\
\hline Neptunium-238 & $<3.410 \mathrm{E}-05$ & $\mu \mathrm{Ci} /$ Sample \\
\hline Neptunium-239 & $<2.075 \mathrm{E}-05$ & $\mu \mathrm{Ci} /$ Sample \\
\hline Plutonium-239 & $<6.872 \mathrm{E}-02$ & $\mu \mathrm{Ci} /$ Sample \\
\hline Americium-241 & $<5.502 \mathrm{E}-05$ & $\mu \mathrm{Ci} /$ Sample \\
\hline Americium-243 & $<1.613 \mathrm{E}-05$ & $\mu \mathrm{Ci} /$ Sample \\
\hline
\end{tabular}


Customer ID: 221T-99-033 Lab Sample \#: S99TP00059
Sample Date : 06/10/1999 11:00

Recv. Date : 06/28/1999 09:07

\begin{tabular}{|c|c|c|}
\hline PARAMETER & RESULTS & UNITS \\
\hline $\begin{array}{l}\text { EnvAlohalBeta } \\
\text { Total Alpha (Each) }\end{array}$ & 8.55E-07 & uCi/Sample \\
\hline Alpha Relative \% Counting Error & $6.92 E+01$ & $\%$ Cl. Error \\
\hline Total Beta (Each) & $2.90 E-06$ & UCi/Sample \\
\hline Beta Relative \% Counting Error & $5.45 E+01$ & $\%$ Ct. Error \\
\hline $\begin{array}{l}\text { GEA Large Librany Method } \\
\text { Berylitum-7 }\end{array}$ & $<7.149$ E-05 & $\mu \mathrm{Ci} / \mathrm{Sample}$ \\
\hline Sodium-22 & $<1.032 E-05$ & $\mu$ Ci/Sample \\
\hline Sodium-24 & $<9.780 \mathrm{E}-06$ & $\mu \mathrm{Ci} /$ Sample \\
\hline Potassium-40 & $<3.867$ E-04 & $\mu \mathrm{Ci} / \mathrm{Sample}$ \\
\hline Argon-41 & $<1.546 E-05$ & HCi/Sample \\
\hline Scandium-46 & $<1.343 E-05$ & HCi/Sample \\
\hline Chromium-51 & $<6.299 E-05$ & $\mu$ Ci/Sample \\
\hline Manganese-54 & $<8.694$ E-06 & $\mu \mathrm{Ci} /$ Sample \\
\hline Cobalt-56 & $<8.917 E-06$ & $\mu$ Ci/Sample \\
\hline Cobalt-57 & $<5.050 E-06$ & HCi/Sample \\
\hline Cobalt-58 & $<8.547 \mathrm{E}-06$ & $\mu$ Ci/Sample \\
\hline Iron-59 & $<1.806 E-05$ & $\mu \mathrm{Ci} /$ Sample \\
\hline Cobalt- 60 & $<9.698 \mathrm{E}-06$ & $\mu \mathrm{Ci} /$ Sample \\
\hline Zinc-65 & $<1.950 E-05$ & $\mu \mathrm{Ci} /$ Sample \\
\hline Selenium-75 & $<9.846$ E-06 & $\mu$ Ci/Sample \\
\hline Krypton-85 & $<2.099 E-03$ & \\
\hline Strontium-85 & $<9.475 E-06$ & $\mu \mathrm{Ci} / \mathrm{Sample}$ \\
\hline Yttrium-88 & $<7.386 \mathrm{E}-06$ & $\mu \mathrm{Ci} /$ Sample \\
\hline Yttrium-91 & $<3.646 \mathrm{E}-03$ & $\mu \mathrm{Ci} /$ Sample \\
\hline Niobium-94 & $<8.681 E-06$ & $\mu \mathrm{Ci} /$ Sample \\
\hline Zirconium/Niobium-95 & $<3.923 E-05$ & $\mu$ Ci/Sample \\
\hline Ruthenium-103 & $<8.076$ E-06 & $\mu \mathrm{Ci} / \mathrm{Sample}$ \\
\hline Ruthenium/Rhodium-106 & $<1.585 \mathrm{E}-04$ & $\mu \mathrm{Ci} /$ Sample \\
\hline Silver-108m & $<9.508 \mathrm{E}-06$ & $\mu \mathrm{Ci} / \mathrm{Sample}$ \\
\hline Cadmium-109 & $<1.657 E-04$ & $\mu \mathrm{Ci} /$ Sample \\
\hline Silver-110m & $<8.809 \mathrm{E}-06$ & $\mu \mathrm{Ci} /$ Sample \\
\hline Tin-113 & $<1.083 E-05$ & HCi/Sample \\
\hline Tellurium-123m & $<5.412 \mathrm{E}-06$ & $\mu$ Ci/Sample \\
\hline Antimony-124 & $<7.923 E-06$ & $\mu \mathrm{Ci} / \mathrm{Sample}$ \\
\hline Antimony-125 & $<2.444 \mathrm{E}-05$ & HCi/Sample \\
\hline Tellurium- $125 \mathrm{~m}$ & $<1.581 \mathrm{E}-03$ & $\mu \mathrm{Ci} /$ Sample \\
\hline lodine-129 & $<5.278 \mathrm{E}-03$ & $\mathrm{MCi} /$ Sample \\
\hline lodine-131 & $<8.338 \mathrm{E}-06$ & $\mu \mathrm{Ci} /$ Sample \\
\hline Xenon-131m & $<2.366 \mathrm{E}-04$ & $\mu \mathrm{Ci} /$ Sample \\
\hline Barium-133 & $<1.030$ E-05 & ICi/Sample \\
\hline Cesium-134 & $<7.937 \mathrm{E}-06$ & $\mu \mathrm{Ci} /$ Sample \\
\hline Cesium-136 & $<8.306 \mathrm{E}-06$ & $\mu \mathrm{Ci} /$ Sample \\
\hline Cesium-137 & $<1.453 E-05$ & $\mu \mathrm{Ci} / \mathrm{Sample}$ \\
\hline Cesium-138 & $<3.839$ E-05 & $\mathrm{CCi} /$ Sample \\
\hline
\end{tabular}


Customer ID: 221T-99-033 (Continued)

Lab Sample \#: S99TP00059

\begin{tabular}{|l|c|c|}
\hline Cerium-139 & $<5.675 \mathrm{E}-06$ & $\mu \mathrm{Ci} /$ Sample \\
\hline Barium-140 & $<3.203 \mathrm{E}-05$ & $\mu \mathrm{Ci} /$ Sample \\
\hline Lanthanum-140 & $<9.214 \mathrm{E}-06$ & $\mu \mathrm{Ci} /$ Sample \\
\hline Cerium-141 & $<9.257 \mathrm{E}-06$ & $\mu \mathrm{Ci} /$ Sample \\
\hline Cerium-144 & $<3.911 \mathrm{E}-05$ & $\mu \mathrm{Ci} /$ Sample \\
\hline Cerium/Praseodymium-144 & $<7.813 \mathrm{E}-05$ & $\mu \mathrm{Ci} /$ Sample \\
\hline Europium-152 & $<1.533 \mathrm{E}-05$ & $\mu \mathrm{Ci} /$ Sample \\
\hline Europium-154 & $<2.999 \mathrm{E}-05$ & $\mu \mathrm{Ci} /$ Sample \\
\hline Europium-155 & $<1.931 \mathrm{E}-05$ & $\mu \mathrm{Ci} /$ Sample \\
\hline Hafnium-181 & $<9.175 \mathrm{E}-06$ & $\mu \mathrm{Ci} /$ Sample \\
\hline Tantalum-182 & $<2.894 \mathrm{E}-05$ & $\mu \mathrm{Ci} /$ Sample \\
\hline Mercury-203 & $<7.618 \mathrm{E}-06$ & $\mu \mathrm{Ci} /$ Sample \\
\hline Bismuth-207 & $<8.207 \mathrm{E}-06$ & $\mu \mathrm{Ci} /$ Sample \\
\hline Thallium-208 & $<9.697 \mathrm{E}-05$ & $\mu \mathrm{Ci} /$ Sample \\
\hline Lead-210 & $<2.243 \mathrm{E}-03$ & $\mu \mathrm{Ci} /$ Sample \\
\hline Bismuth-212 & $<1.385 \mathrm{E}-04$ & $\mu \mathrm{Ci} /$ Sample \\
\hline Lead-212 & $<1.484 \mathrm{E}-05$ & $\mu \mathrm{Ci} /$ Sample \\
\hline Bismuth-214 & $<2.682 \mathrm{E}-05$ & $\mu \mathrm{Ci} /$ Sample \\
\hline Lead-214 & $<9.036 \mathrm{E}-05$ & $\mu \mathrm{Ci} /$ Sample \\
\hline Radium-224 & $<1.559 \mathrm{E}-04$ & $\mu \mathrm{Ci} /$ Sample \\
\hline Radium-226 & $<1.485 \mathrm{E}-04$ & $\mu \mathrm{Ci} /$ Sample \\
\hline Actinium-228 & $<4.972 \mathrm{E}-05$ & $\mu \mathrm{Ci} /$ Sample \\
\hline Thorium-228 & $<5.509 \mathrm{E}-04$ & $\mu \mathrm{Ci} /$ Sample \\
\hline Thorium-229 & $<2.398 \mathrm{E}-05$ & $\mu \mathrm{Ci} /$ Sample \\
\hline Uranium-232 & $<1.112 \mathrm{E}-02$ & $\mu \mathrm{Ci} /$ Sample \\
\hline Protactinum-233 & $<1.607 \mathrm{E}-05$ & $\mu \mathrm{Ci} /$ Sample \\
\hline Uranium/Thorium-233 & $<5.263 \mathrm{E}-03$ & $\mu \mathrm{Ci} /$ Sample \\
\hline Protactinium-234m & $<1.563 \mathrm{E}-03$ & $\mu \mathrm{Ci} /$ Sample \\
\hline Thorium-234 & $<4.023 \mathrm{E}-04$ & $\mu \mathrm{Ci} /$ Sample \\
\hline Uranium-235 & $<8.937 \mathrm{E}-06$ & $\mu \mathrm{Ci} /$ Sample \\
\hline Neptunium-237 & $<5.132 \mathrm{E}-05$ & $\mu \mathrm{Ci} /$ Sample \\
\hline Uranium-237 & $<1.871 \mathrm{E}-05$ & $\mu \mathrm{Ci} /$ Sample \\
\hline Neptunium-238 & $<3.476 \mathrm{E}-05$ & $\mu \mathrm{Ci} /$ Sample \\
\hline Neplunium-239 & $<1.969 \mathrm{E}-05$ & $\mu \mathrm{Ci} /$ Sample \\
\hline Plutonium-239 & $<6.688 \mathrm{E}-02$ & $\mu \mathrm{Ci} /$ Sample \\
\hline Americium-241 & $<5.492 \mathrm{E}-05$ & $\mu \mathrm{Ci} /$ Sample \\
\hline Americium-243 & $<1.590 \mathrm{E}-05$ & $\mu \mathrm{Ci} /$ Sample \\
\hline
\end{tabular}


Customer 1D: 221T-99-034 Lab Sample \#: S99TP00060
Sample Date : 06/24/1999 15:30

Recv. Date : 06/28/1999 09:07

\begin{tabular}{|c|c|c|}
\hline PARAMETER & RESULTS & UNITS \\
\hline $\begin{array}{l}\text { Eny Alpha/Beta } \\
\text { Total Alpha (Each) }\end{array}$ & $1.22 E-06$ & uCi/Sample \\
\hline Alpha Relative \% Counting Error & $5.40 \mathrm{E}+01$ & $\%$ C. Error \\
\hline Total Beta (Each) & 9.19E-06 & uCi/Sample \\
\hline Beta Relative \% Counting Error & $1.99 E+01$ & $\%$ Cl. Error \\
\hline $\begin{array}{l}\text { GEA Large Librari Methed } \\
\text { Beryllium-7 }\end{array}$ & $<7.185 E-05$ & $\mu \mathrm{Ci} /$ Sample \\
\hline Sodium-22 & $<1.064$ E-05 & $\mu \mathrm{Ci} /$ Sample \\
\hline Sodium-24 & $<9.310 \mathrm{E}-06$ & $\mu \mathrm{Ci} / \mathrm{Sample}$ \\
\hline Potassium-40 & $<3.852 \mathrm{E}-04$ & $\mu \mathrm{Ci} /$ Sample \\
\hline Argon-41 & $<1.516 \mathrm{E}-05$ & $\mu \mathrm{Ci} / \mathrm{Sample}$ \\
\hline Scandium-46 & $<1.368 E-05$ & $\mu \mathrm{Ci} / \mathrm{Sample}$ \\
\hline Chromium-51 & $<6.396 \mathrm{E}-05$ & $\mu \mathrm{Ci} / \mathrm{Sample}$ \\
\hline Manganese-54 & $<9.028 \mathrm{E}-06$ & $\mu \mathrm{Ci} /$ Sample \\
\hline Cobalt-56 & $<9.553 \mathrm{E}-06$ & $\mu \mathrm{Ci} /$ Sample \\
\hline Cobalt-57 & $<5.060 \mathrm{E}-06$ & $\mathrm{C}$ Ci/Sample \\
\hline Cobalt-58 & $<8.400$ E-06 & $\mu \mathrm{Ci} /$ Sample \\
\hline Iron-59 & $<1.771 \mathrm{E}-05$ & $\mu \mathrm{Ci} /$ Sample \\
\hline Cobalt-60 & $<9.083 E-06$ & $\mu \mathrm{Ci} /$ Sample \\
\hline Zinc-65 & $<2.056 \mathrm{E}-05$ & $\mu \mathrm{Ci} /$ Sample \\
\hline Selenium-75 & $<9.819 E-06$ & $\mu \mathrm{Ci} /$ Sample \\
\hline Krypton-85 & $<2.059 E-03$ & $\mu$ Ci/Sample \\
\hline Strontium-85 & $<9.292 E-06$ & $\mu \mathrm{Ci} /$ Sample \\
\hline Yttrium-88 & $<7.445 \mathrm{E}-06$ & $\mu \mathrm{Ci} /$ Sample \\
\hline Yttrium-91 & $<3.740$ E-03 & $\mu \mathrm{Ci} /$ Sample \\
\hline Niobium-94 & $<9.247 \mathrm{E}-06$ & $\mu \mathrm{Ci} /$ Sample \\
\hline Zirconium/Niobium-95 & $<3.799 \mathrm{E}-05$ & $\mu \mathrm{Ci} / \mathrm{Sample}$ \\
\hline Ruthenium-103 & $<8.040$ E-06 & 드/Sample \\
\hline Ruthenium/Rhodium-106 & $<1.577 \mathrm{E}-04$ & $\mu \mathrm{Ci} / \mathrm{Sample}$ \\
\hline Silver-108m & $<8.899 \mathrm{E}-06$ & $\mu \mathrm{Ci} /$ Sample \\
\hline Cadmium-109 & $<1.635 \mathrm{E}-04$ & $\mu \mathrm{Ci} / \mathrm{Sample}$ \\
\hline Silver-110m & $<8.503 \mathrm{E}-06$ & $\mu \mathrm{Ci} /$ Sample \\
\hline $\operatorname{Tin}-113$ & $<1.091 \mathrm{E}-05$ & $\mu \mathrm{Ci} /$ Sample \\
\hline Tellurium-123m & $<5.411 E-06$ & $\mu \mathrm{Ci} / \mathrm{Sample}$ \\
\hline Antimony-124 & $<7.847$ E-06 & $\mu \mathrm{Ci} /$ Sample \\
\hline Antimony-125 & $<2.366 \mathrm{E}-05$ & ${ }$ Ci/Sample \\
\hline Tellurium-125m & $<1.564 \mathrm{E}-03$ & $\mu \mathrm{Ci} / \mathrm{Sample}$ \\
\hline lodine-129 & $<5.359 E-03$ & $\mu \mathrm{Ci} /$ Sample \\
\hline lodine-131 & $<8.091 E-06$ & $\mu \mathrm{Ci} /$ Sample \\
\hline Xenon-131m & $<2.343 \mathrm{E}-04$ & $\mu \mathrm{Ci} /$ Sample \\
\hline Barium-133 & $<1.053 \mathrm{E}-05$ & $\mu \mathrm{Ci} /$ Sample \\
\hline Cesium-134 & $<8.028 \mathrm{E}-06$ & $\mu \mathrm{Ci} /$ Sample \\
\hline Cesium-136 & $<8.977 \mathrm{E}-06$ & $\mu \mathrm{Ci} /$ Sample \\
\hline Cesium-137 & $<1.451 E-05$ & $\mu \mathrm{Ci} /$ Sample \\
\hline Cesium-138 & $<4.086 \mathrm{E}-05$ & $\mu \mathrm{Ci} /$ Sample \\
\hline
\end{tabular}


Customer ID: 221T-99-034 (Continued)

Lab Sample \#: S99TP00060

\begin{tabular}{|c|c|c|}
\hline Cerium-139 & $<5.696 \mathrm{E}-06$ & HCi/Sample \\
\hline Barium-140 & $<3.121 \mathrm{E}-05$ & HCi/Sample \\
\hline Lanthanum-140 & $<8.777$ E-06 & $\mu \mathrm{Ci} /$ Sample \\
\hline Cerium-141 & $<9.154 E-06$ & $\mu \mathrm{Ci} /$ Sample \\
\hline Cerium-144 & $<4.004 E-05$ & $\mu \mathrm{Ci} /$ Sample \\
\hline Cerium/Praseodymium-144 & $<8.002 E-05$ & $\mu \mathrm{Ci} / \mathrm{Sample}$ \\
\hline Europium-152 & $<1.532 E-05$ & $\mu \mathrm{Ci} /$ Sample \\
\hline Europium-154 & $<3.095 \mathrm{E}-05$ & $\mu \mathrm{Ci} /$ Sample \\
\hline Europium-155 & $<1.903 E-05$ & $\mu \mathrm{Ci} /$ Sample \\
\hline Hafnium-181 & $<9.101 \mathrm{E}-06$ & $\mu \mathrm{Ci} / \mathrm{Sample}$ \\
\hline Tantalum-182 & $<2.843 E-05$ & $\mu \mathrm{Ci} /$ Sample \\
\hline Mercury-203 & $<7.307 E-06$ & $\mu \mathrm{Ci} /$ Sample \\
\hline Bismuth-207 & $<8.062 \mathrm{E}-06$ & $\mu \mathrm{Ci} / \mathrm{Sample}$ \\
\hline Thallium-208 & $<9.518 E-05$ & $\mu \mathrm{Ci} /$ Sample \\
\hline Lead-210 & $<2.223 E-03$ & $\mu \mathrm{Ci} /$ Sample \\
\hline Bismuth-212 & $<1.419 \mathrm{E}-04$ & $\mu \mathrm{Ci} /$ Sample \\
\hline Lead-212 & $<1.474 \mathrm{E}-05$ & $\mu \mathrm{Ci} /$ Sample \\
\hline Bismuth-214 & $<2.589 E-05$ & $\mu \mathrm{Ci} /$ Sample \\
\hline Lead-214 & $<8.537$ E-05 & $\mu \mathrm{Ci} /$ Sample \\
\hline Radium-224 & $<1.529$ E-04 & $\mu \mathrm{Ci} / \mathrm{Sample}$ \\
\hline Radium-226 & $<1.447 E-04$ & $\mu \mathrm{Ci} / \mathrm{Sample}$ \\
\hline Actinium-228 & $<4.872 E-05$ & $\mu$ Ci/Sample \\
\hline Thorium-228 & $<5.374 E-04$ & $\mu \mathrm{Ci} / \mathrm{Sample}$ \\
\hline Thorium-229 & $<2.370$ E-05 & $\mu \mathrm{Ci} /$ Sample \\
\hline Uranium-232 & $<1.074 \mathrm{E}-02$ & $\mu \mathrm{Ci} / \mathrm{Sample}$ \\
\hline Protactinum-233 & $<1.629 E-05$ & $\mu \mathrm{Ci} /$ Sample \\
\hline Uranium/Thorium-233 & $<5.180 \mathrm{E}-03$ & $\mu \mathrm{Ci} / \mathrm{Sample}$ \\
\hline Prolactinium-234m & $<1.509 \mathrm{E}-03$ & $\mu \mathrm{Ci} / \mathrm{Sample}$ \\
\hline Thorium-234 & $<3.987$ E-04 & $\mu \mathrm{Ci} /$ Sample \\
\hline Uranium-235 & $<8.820 \mathrm{E}-06$ & $\mu \mathrm{Ci} /$ Sample \\
\hline Neptunium-237 & $<5.049 \mathrm{E}-05$ & $\mu \mathrm{Ci} / \mathrm{Sample}$ \\
\hline Uranium-237 & $<1.856 \mathrm{E}-05$ & $\mu \mathrm{Ci} /$ Sample \\
\hline Neptunium-238 & $<3.333 E-05$ & $\mu \mathrm{Ci} / \mathrm{S}$ ample \\
\hline Neptunium-239 & $<2.013 E-05$ & $\mu \mathrm{Ci} / \mathrm{Sample}$ \\
\hline Plutonium-239 & $<6.892 \mathrm{E}-02$ & $\mu \mathrm{Ci} /$ Sample \\
\hline Americium-241 & $<5.533 \mathrm{E}-05$ & $\mu \mathrm{Ci} /$ Sample \\
\hline Americium-243 & $<1.612$ E-05 & $\mu$ Ci/Sample \\
\hline
\end{tabular}


Customer ID: 221T-99-035 BLANK Lab Sample \#: N/A
Sample Date : 06/24/1999 11:00

Recv. Date : 06/28/1999 09:07

\begin{tabular}{|c|c|c|}
\hline PARAMETER & RESULTS & UNITS \\
\hline $\begin{array}{l}\text { EnvAlpha/Beta } \\
\text { Total Alpha (Each) }\end{array}$ & 3.63E-07 & $\mu \mathrm{Ci} /$ Sample \\
\hline Alpha Relative \% Counting Error & $1.33 E+02$ & $\%$ Ct. Error \\
\hline Total Beta (Each) & 4.47E-06 & $\mu \mathrm{Ci} /$ Sample \\
\hline Beta Relative \% Counting Error & $3.68 \mathrm{E}+01$ & $\%$ Cl. Error \\
\hline $\begin{array}{l}\text { GEA Laroe Library Method } \\
\text { Beryllium-7 }\end{array}$ & $<7.392 \mathrm{E}-05$ & «Ci/Sample \\
\hline Sodium-22 & $<1.052$ E-05 & $\mu \mathrm{Ci} /$ Sample \\
\hline Sodium-24 & $<9.921 \mathrm{E}-06$ & HCi/Sample \\
\hline Potassium -40 & $<3.875 \mathrm{E}-04$ & $\mu \mathrm{Ci} / \mathrm{Sample}$ \\
\hline Argon-41 & $<1.508 \mathrm{E}-05$ & HCi/Sample \\
\hline Scandium-46 & $<1.393 E-05$ & $\mu \mathrm{Ci} /$ Sample \\
\hline Chromium-51 & $<6.388 \mathrm{E}-05$ & $\mu \mathrm{Ci} /$ Sample \\
\hline Manganese-54 & $<8.594 E-06$ & $\mu \mathrm{Ci} /$ Sample \\
\hline Cobalt-56 & $<9.156 E-06$ & $\mu \mathrm{Cl} / \mathrm{S}$ ample \\
\hline Cobalt-57 & $<5.148 E-06$ & $\mu \mathrm{Ci} /$ Sample \\
\hline Cobalt-58 & $<8.859 E-06$ & $\mu \mathrm{Ci} /$ Sample \\
\hline Iron-59 & $<1.863 E-05$ & $\mathrm{Ci} / \mathrm{S}$ Sample \\
\hline Cobalt-60 & $<9.746 E-06$ & $\mu \mathrm{Ci} /$ Sample \\
\hline Zinc-65 & $<2.132 \mathrm{E}-05$ & $1 \mathrm{Ci} /$ Sample \\
\hline Selenium-75 & $<1.004 E-05$ & $\mu \mathrm{Ci} /$ Sample \\
\hline Krypton-85 & $<2.043 E-03$ & $\mu \mathrm{Ci} / \mathrm{Sample}$ \\
\hline Strontium-85 & $<9.217 \mathrm{E}-06$ & $\mu \mathrm{Ci} /$ Sample \\
\hline Yttrium-88 & $<7.753 \mathrm{E}-06$ & HCi/Sample \\
\hline Yttrium-91 & $<3.796 E-03$ & $\mu \mathrm{Ci} /$ Sample \\
\hline Niobium-94 & $<9.026 E-06$ & $\mu \mathrm{Ci} /$ Sample \\
\hline Zirconium/Niobium-95 & $<4.115 E-05$ & $\mu \mathrm{Ci} / \mathrm{Sample}$ \\
\hline Ruthenium-103 & $<8.343 E-06$ & $\mu \mathrm{Ci} /$ Sample \\
\hline Ruthenium/Rhodium-106 & $<1.538 \mathrm{E}-04$ & $\mu \mathrm{Ci} / \mathrm{Sample}$ \\
\hline Silver-108m & $<9.133 E-06$ & $\mu \mathrm{Ci} /$ Sample \\
\hline Cadmium-109 & $<1.682 E-04$ & $\mu \mathrm{Ci} /$ Sample \\
\hline Silver-110m & $<8.491 E-06$ & $\mu \mathrm{Ci} /$ Sample \\
\hline Tin-113 & $<1.065 \mathrm{E}-05$ & $\mu \mathrm{Ci} /$ Sample \\
\hline Tellurium-123m & $<5.423 \mathrm{E}-06$ & $\mu \mathrm{Ci} /$ Sample \\
\hline Antimony-124 & $<8.370$ E-06 & $\mu$ Ci/Sample \\
\hline Antimony-125 & $<2.443 E-05$ & \\
\hline Tellurium-125m & $<1.627 \mathrm{E}-03$ & $\mu \mathrm{Ci} /$ Sample \\
\hline lodine-129 & $<5.629 E-03$ & HCi/Sample \\
\hline Iodine-131 & $<8.551 E-06$ & $\mu \mathrm{Ci} / \mathrm{Sample}$ \\
\hline Xenon-131m & $<2.371 E-04$ & $\mu \mathrm{Ci} /$ Sample \\
\hline Barium-133 & $<1.042$ E-05 & $\mu \mathrm{Ci} / \mathrm{Sample}$ \\
\hline Cesium-134 & $<8.479 E-06$ & $\mu \mathrm{Ci} /$ Sample \\
\hline Cesium-136 & $<9.043 E-06$ & $\mu \mathrm{Ci} /$ Sample \\
\hline Cesium-137 & $<1.483 \mathrm{E}-05$ & $\mu \mathrm{Ci} /$ Sample \\
\hline Cesium-138 & $<3.762$ E-05 & $\mu \mathrm{Ci} /$ Sample \\
\hline
\end{tabular}


Customer ID: 221T-99-035 BLANK (Continued) Lab Sample \#: N/A

\begin{tabular}{|c|c|c|}
\hline Cerium-139 & $<5.828 \mathrm{E}-06$ & $\mu \mathrm{Ci} / \mathrm{Sample}$ \\
\hline Barium-140 & $<3.184 \mathrm{E}-05$ & $\mu \mathrm{Ci} / \mathrm{Sample}$ \\
\hline Lanthanum-140 & $<8.906 \mathrm{E}-06$ & $\mu \mathrm{Ci} /$ Sample \\
\hline Cerium-141 & $<8.988 \mathrm{E}-06$ & $\mu \mathrm{Ci} / \mathrm{Sample}$ \\
\hline Cerium-144 & $<3.960$ E-05 & $\mu \mathrm{Ci} / \mathrm{Sample}$ \\
\hline Cerium/Praseodymium-144 & $<7.913 E-05$ & $\mu \mathrm{Ci} /$ Sample \\
\hline Europium-152 & $<1.576 \mathrm{E}-05$ & $\mu \mathrm{Ci} /$ Sample \\
\hline Europium-154 & $<.3 .057 \mathrm{E}-05$ & $\mu \mathrm{Ci} /$ Sample \\
\hline Europium-155 & $<1.955 E-05$ & $\mu \mathrm{Ci} /$ Sample \\
\hline Hafnium-181 & $<9.038 \mathrm{E}-06$ & $\mu \mathrm{Ci} /$ Sample \\
\hline Tantalum-182 & $<2.809 E-05$ & $\mu \mathrm{Ci} /$ Sample \\
\hline Mercury-203 & $<7.418 E-06$ & $\mu \mathrm{Ci} /$ Sample \\
\hline Bismuth-207 & $<8.026 \mathrm{E}-06$ & $\mu \mathrm{Ci} /$ Sample \\
\hline Thallium-208 & $<9.687 \mathrm{E}-05$ & $\mu \mathrm{Ci} /$ Sample \\
\hline Lead-210 & $<2.371 \mathrm{E}-03$ & $\mu \mathrm{Ci} / \mathrm{Sample}$ \\
\hline Bismuth-212 & $<1.498 \mathrm{E}-04$ & HCi/Sample \\
\hline Lead-212 & $<1.485 \mathrm{E}-05$ & $\mu \mathrm{Ci} /$ Sample \\
\hline Bismuth-214 & $<2.758 \mathrm{E}-05$ & $\mu \mathrm{Ci} /$ Sample \\
\hline Lead-214 & $<9.469 E-05$ & $\mu \mathrm{Ci} /$ Sample \\
\hline Radium-224 & $<1.549$ E-04 & $\mu \mathrm{Ci} /$ Sample \\
\hline Radium-226 & $<1.454 \mathrm{E}-04$ & $\mu \mathrm{Ci} /$ Sample \\
\hline Actinium-228 & $<5.063 E-05$ & $\mu$ Ci/Sample \\
\hline Thorium-228 & $<5.543 E-04$ & $\mu \mathrm{Ci} /$ Sample \\
\hline Thorium-229 & $<2.429 \mathrm{E}-05$ & $\mu \mathrm{Ci} /$ Sample \\
\hline Uranium-232 & $<1.145 E-02$ & $\mu \mathrm{Ci} / \mathrm{Sample}$ \\
\hline Protactinum-233 & $<1.670 \mathrm{E}-05$ & $\mu \mathrm{Ci} /$ Sample \\
\hline Uranium/Thorium-233 & $<5.110$ E-03 & $\mu \mathrm{Ci} /$ Sample \\
\hline Protactinium-234m & $<1.546 \mathrm{E}-03$ & $\mu \mathrm{Ci} / \mathrm{Sample}$ \\
\hline Thorium-234 & $<4.021 E-04$ & $\mu \mathrm{Ci} /$ Sample \\
\hline Uranium-235 & $<8.902 E-06$ & $\mu \mathrm{Ci} /$ Sample \\
\hline Neptunium-237 & $<5.204 \mathrm{E}-05$ & $\mu \mathrm{Ci} /$ Sample \\
\hline Uranium-237 & $<1.942 E-05$ & $\mu \mathrm{Ci} / \mathrm{Sample}$ \\
\hline Neptunium-238 & $<3.416 E-05$ & $\mu \mathrm{Ci} / \mathrm{Sample}$ \\
\hline Neptunium-239 & $<2.071 E-05$ & $\mu \mathrm{Ci} /$ Sample \\
\hline Plutonium-239 & $<6.766 \mathrm{E}-02$ & $\mu \mathrm{Ci} / \mathrm{Sample}$ \\
\hline Americium-241 & $<5.559 E-05$ & $\mu \mathrm{Ci} /$ Sample \\
\hline Americium-243 & $<1.643 E-05$ & $\mu \mathrm{Ci} /$ Sample \\
\hline
\end{tabular}


Customer ID: 221T-99-035 SPIKE Sample Date : 06/24/1999 11:00 Lab Sample \#: S99TP00062

Recv. Date : 06/28/1999 09:07

\begin{tabular}{|l|c|l|}
\hline PARAMETER & RESULTS & \multicolumn{1}{c|}{ UNITS } \\
\hline EnvAlpha/Beta & $3.36 E-03$ & UCi/Sample \\
\hline Total Alpha (Each) & $8.39 E-01$ & $\%$ Ct. Error \\
\hline Alpha Relative \% Counting Error & $1.39 E-02$ & UCi/Sample \\
\hline Total Beta (Each) & $3.11 \mathrm{E}-01$ & $\%$ Ct. Error \\
\hline Beta Relative \% Counting Error & & \\
\hline Calculated Spike Recoveries* & & \% Recovery \\
\hline Total Alpha & $\mathbf{8 8 . 2}$ & \% Recovery \\
\hline Total Beta & 103.6 &
\end{tabular}

-Spike added to the sample digest 
DOE/RL-99-60, Rev. 0

$09 / 99$

\section{APPENDIX B}

\section{CALCULATED RELEASE FRACTIONS}




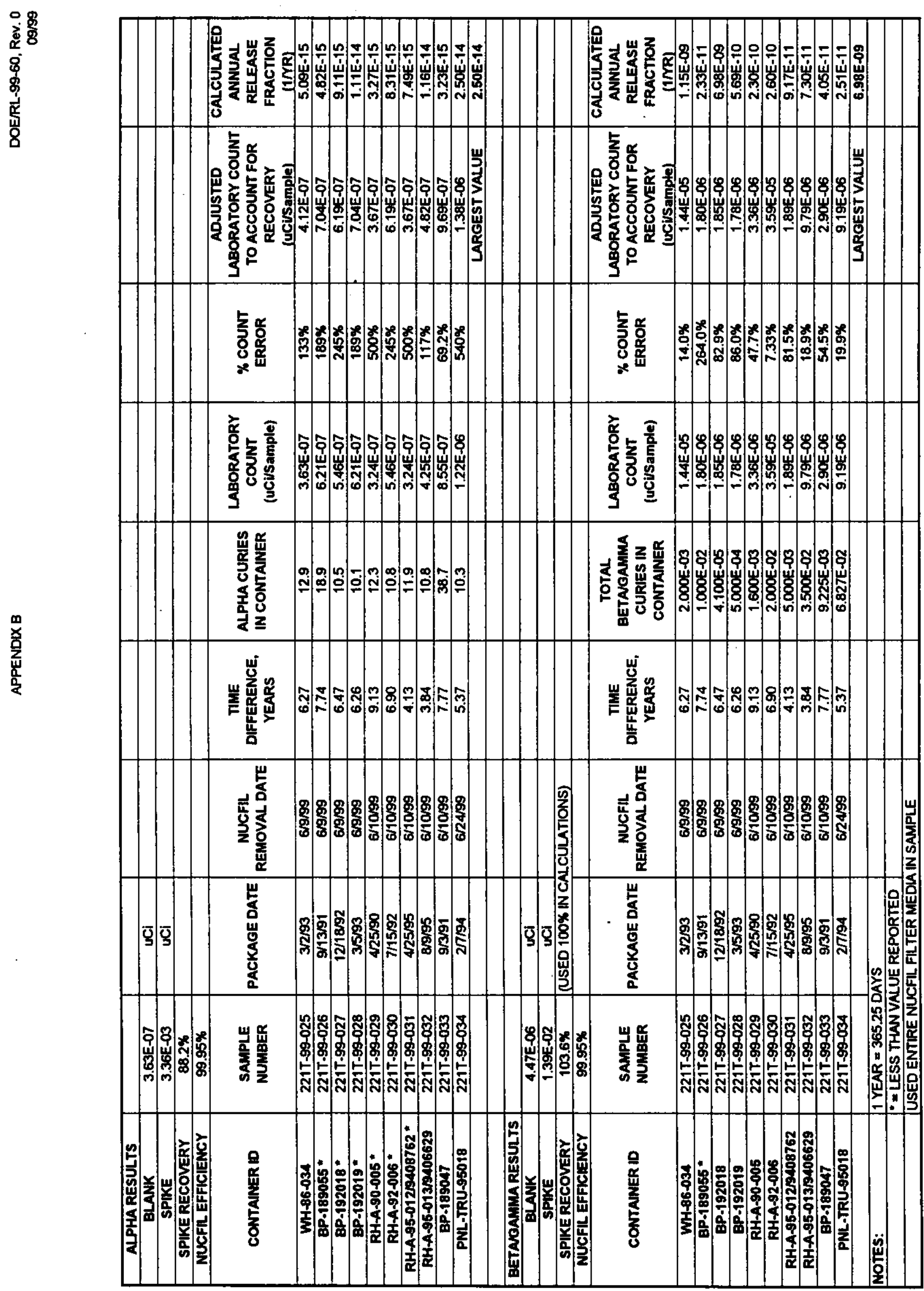




\section{DISTRIBUTION}

J. Leitch

United States Environmental Protection Agency

Region 10

1200 Sixth Avenue

Seattle, Washington 98101

A. W. Conklin

Washington State Department of Health

7171 Cleanwater Lane, Building 5

Olympia, Washington 98504

R. S. Acselrod

Washington State Department of Health

PMB 385

2839 W. Kennewick, Avenue

Kennewick, Washington 99336

P. J. Martell

Washington State Department of Health

PMB 385

2839 W. Kennewick, Avenue

Kennewick, Washington 99336

J. W. Schmidt

Washington State Department of Health

PMB 385

2839 W. Kennewick, Avenue

Kennewick, Washington 99336

D. A. Dunning

Oregon Office of Energy

625 Marrian Street N.E.

Suite 1

Salem, Oregon 97301

J. Wilkinson

Confederated Tribes of the Umatilla Indian Nation

P. O. Box 638

Pendleton, Oregon 97801

P. Sobotta

Nez Perce Tribe

P. O. Box 365

Lapwai, Idaho 93540 


\section{DISTRIBUTION}

MSIN

R. Jim, Manager

Environmental Restoration/Waste Management Program Yakima Indian Nation

P. O. Box 151

Toppenish, Washington 98948

U.S. Department of Energy, Richland Operations Office

C. J. Bosted

S7-41

J. E. Rasmussen

A5-15

G. L. Sinton

S7-55

S. D. Stites

A5-15

Public Reading Room

$\mathrm{H} 2-53$

Fluor Daniel Hanford, Inc.

W. D. Adair

AO-22

J. A. Bates

AO-22

A. G. Miskho

H6-06

J. D. Williams

H6-06

Pacific Northwest National Laboratory

Hanford Technical Library

P8-55

Waste Management Federal Services Hanford, Inc.

R. B. Barmettlor

T4-04

B. M. Barnes

T4-04

B. L. Curn

G1-29

W. E. Davis

G1-29

L. P. Diediker

G1-29

E. M. Greager

G1-29

J. S. Hill

G1-29

R. E. Johnson

G1-29

L. D. Kamberg

T4-04

J. J. Luke

G1-29

L. L. Powers

G1-27

D. L. Renberger

H6-32

G. T. Wells

Gl-29

L. F. Willis

G1-32

Lockheed Martin Services, Inc.

Central Files

B1-07

DPC

H6-08

EDMC (2)

H6-08 